\title{
Experimental study of the turbulent boundary layer in acceleration-skewed oscillatory flow
}

\author{
Dominic A. van der $\mathrm{A}^{1} \dagger$, Tom O’Donoghue ${ }^{1}$, \\ Alan G. Davies ${ }^{2}$ and Jan S. Ribberink ${ }^{3}$ \\ ${ }^{1}$ School of Engineering, University of Aberdeen, Aberdeen AB24 3UE, UK \\ ${ }^{2}$ School of Ocean Sciences, Bangor University, Menai Bridge, Anglesey LL59 5AB, UK \\ ${ }^{3}$ Department of Water Engineering and Management, University of Twente, Enschede, PO Box 217, \\ 7500 AE, The Netherlands
}

(Received 11 July 2010; revised 26 May 2011; accepted 6 July 2011; first published online 6 September 2011)

Experiments have been conducted in a large oscillatory flow tunnel to investigate the effects of acceleration skewness on oscillatory boundary layer flow over fixed beds. As well as enabling experimental investigation of the effects of acceleration skewness, the new experiments add substantially to the relatively few existing detailed experimental datasets for oscillatory boundary layer flow conditions that correspond to full-scale sea wave conditions. Two types of bed roughness and a range of highReynolds-number, $\operatorname{Re} \sim O\left(10^{6}\right)$, oscillatory flow conditions, varying from sinusoidal to highly acceleration-skewed, are considered. Results show the structure of the intrawave velocity profile, the time-averaged residual flow and boundary layer thickness for varying degrees of acceleration skewness, $\beta$. Turbulence intensity measurements from particle image velocimetry (PIV) and laser Doppler anemometry (LDA) show very good agreement. Turbulence intensity and Reynolds stress increase as the flow accelerates after flow reversal, are maximum at around maximum free-stream velocity and decay as the flow decelerates. The intra-wave turbulence depends strongly on $\beta$ but period-averaged turbulent quantities are largely independent of $\beta$. There is generally good agreement between bed shear stress estimates obtained using the loglaw and using the momentum integral equation, and flow acceleration skewness leads to high bed shear stress asymmetry between flow half-cycles. Turbulent Reynolds stress is much less than the shear stress obtained from the momentum integral; analysis of the stress contributors shows that significant phase-averaged vertical velocities exist near the bed throughout the flow cycle, which lead to an additional shear stress, $-\rho \tilde{u} \tilde{w}$; near the bed this stress is at least as large as the turbulent Reynolds stress.

Key words: coastal engineering, geophysical and geological flows, turbulent boundary layers

\section{Introduction}

Sea waves in coastal waters generate near-bed flows with combined velocity and acceleration skewness, with the degree of each skewness type being dependent

$†$ Email address for correspondence: d.a.vandera@abdn.ac.uk 
on wave period, wave height and water depth (Elfrink, Hanes \& Ruessink 2006). Skewness creates asymmetry in bed shear stress, sediment pick-up and sediment flux between the positive and negative flow half-cycles within the wave period, and so plays a primary role in determining the wave-driven net sediment transport. While the effects of velocity skewness on boundary layer flow and sediment transport have been well studied (e.g. Ribberink \& Al-Salem 1994; Davies \& Li 1997), and sediment transport models now incorporate velocity skewness effects to a greater or lesser extent, the effects of acceleration skewness have been much less studied. Waves with high degree of acceleration skewness generate a sawtooth-shaped free-stream velocity time series above the wave boundary layer, with strong asymmetry in flow acceleration between the positive and negative half-cycles. Analytical (Nielsen 1992; Foster, Guenther \& Holman 1999) and numerical (Hsu \& Hanes 2004; GonzalezRodriguez \& Madsen 2007; Fuhrman, Fredsøe \& Sumer 2009) models show that significantly higher bed shear stress is associated with the higher acceleration and that the skewness generates a residual flow in the boundary layer that is against the direction of highest acceleration. The impact on net sediment transport is substantial, as demonstrated by the laboratory experiments of Watanabe \& Sato (2004) and van der A, O'Donoghue \& Ribberink (2010) and by Elgar, Gallagher \& Guza's (2001) field observations of onshore sandbar migration. As a consequence, new sediment transport formulations have recently been proposed to account for so-called 'acceleration effects' (van der A et al. 2010).

Present understanding of oscillatory boundary layers is based on laboratory experiments, including experiments in which beds are oscillated in otherwise still water (Keiller \& Sleath 1976; Krstic \& Fernando 2001) and experiments in smallscale wave flumes (Sleath 1970; Kemp \& Simons 1982, 1983; Cox, Kobayashi \& Okayasu 1996; Mirfenderesk \& Young 2003; Dixen et al. 2008) or oscillatory open channel flumes (Chen et al. 2007). However, the physical limitations of these facilities mean that the amplitude Reynolds number, $R e=u_{0 \max } a / v$ (where $u_{0 \max }$ is the amplitude of the free-stream horizontal velocity, $a$ the amplitude of the freestream water particle excursion and $v$ is the kinematic viscosity), and the relative roughness $a / k_{s}$ (where $k_{s}$ is the equivalent bed roughness) are low and are not representative of conditions under full-scale waves. Much higher $R e$ and $a / k_{s}$ can be achieved by conducting experiments in large oscillatory flow tunnels. Jonsson (1963) and Jonsson \& Carlsen (1976) were among the first to measure boundary layer velocities, but not turbulence, for sinusoidally varying flow over rough beds in an oscillatory flow tunnel at high $R e \sim O\left(10^{6}\right)$, but the most detailed studies are those of Sleath (1987) and Jensen, Sumer \& Fredsøe (1989). Sleath (1987) measured the detailed intra-wave velocities, including turbulence, for a range of bed roughness and sinusoidally varying flows with $R e \cong 3 \times 10^{5}$. His results showed the periodic behaviour in turbulence generation and dissipation, with turbulence being generated at the bed, increasing and diffusing upwards as the flow accelerates and reaching a maximum at around maximum free-stream velocity, before decaying as the flow decelerates. In addition to the turbulence behaviour, Sleath presents phase-averaged velocity profiles, measures of the phase lead and boundary layer thickness and results for shear stress. A particularly significant result is the large difference between bed shear stress estimated from the measured turbulent stresses and bed shear stress estimated via momentum integral applied to the phase-averaged velocity profiles; the difference was not resolvable from Sleath's measurements. Jensen et al. (1989) conducted similarly detailed experiments, for both rough and smooth beds, but for higher $\operatorname{Re} \sim O\left(10^{6}\right)$ corresponding to high-energy wave conditions. In addition to Sleath and Jensen et al., 
other oscillatory flow tunnel experiments have been conducted to study the effect of a sudden change in bed roughness (Fredsøe et al. 1993), vertical oscillatory flow through the bed (Conley \& Inman 1994) and grid-generated turbulence (Fredsøe et al. 2003) on the oscillatory flow. Like Sleath (1987) and Jensen et al. (1989), all of these experiments involved oscillatory flow without acceleration skewness. Sumer, Laursen \& Fredsøe (1993) conducted flow tunnel experiments with sinusoidal flow through a converging-diverging section within the tunnel, thereby generating flows with convective acceleration skewness.

The present paper reports on new experiments conducted in a large oscillatory flow tunnel to investigate the effects of acceleration skewness on oscillatory boundary layer flow, including turbulence and bed shear stress. The experiments involve two types of bed roughness and a range of high-Re oscillatory flow conditions varying from sinusoidal to highly acceleration-skewed. Velocities were measured using particle image velocimetry (PIV), supported by laser Doppler anemometer (LDA) point measurements of velocity at several elevations within the boundary layer. As well as enabling experimental investigation of the effects of acceleration skewness, the new experiments add substantially to the relatively few existing detailed experimental datasets (Sleath 1987; Jensen et al. 1989) for oscillatory boundary layer flow conditions that correspond to full-scale sea wave conditions.

The experimental setup and data processing methods are described in $\S 2$ and the experimental conditions are presented in $\S 3$, showing where they lie in $R e-a / k_{s}$ space compared with previous experiments. The results are presented in three sections as follows. First, phase-averaged velocities are presented in $\S 4$, showing the details of the intra-wave velocity profiles, boundary layer phase lead, boundary layer thickness and near-bed residual. Second, the turbulence measurements are presented in $\S 5$, showing time series and vertical profiles of intra-wave and period-averaged turbulence intensity and Reynolds stress. Finally, shear stress results are presented in $\S 6$ : the intra-wave bed shear stress is determined from a log-law fit and application of the momentum integral equation to the phase-averaged velocity profiles and a detailed analysis is made of the contributors to total shear stress; wave friction factors are determined from the log-law bed-shear stress estimates. The main conclusions are stated in $\S 7$.

\section{Experimental setup}

\subsection{Experimental facility}

The Aberdeen oscillatory flow tunnel (AOFT) is a large laboratory facility in which near-bed horizontal flows, equivalent in period and amplitude to the near-bed flows beneath full-scale waves, can be generated. In tunnels of this kind the flow is driven through a closed rectangular-section conduit. There is no free water surface in the test section, which means that the oscillatory flow differs from that generated by surface waves in having zero vertical orbital motion in the free stream and being horizontally uniform. The AOFT (figure 1) is of U-tube construction with an overall length of $16 \mathrm{~m}, 10 \mathrm{~m}$ of which is a glass-sided rectangular test section, $0.75 \mathrm{~m}$ high and $0.3 \mathrm{~m}$ wide. Open reservoirs at either end of the tunnel accommodate the volume of water displaced by the horizontally driven piston. The $1 \mathrm{~m}$ diameter piston is housed in a circular cylindrical section at one end of the tunnel and is electro-hydraulically controlled within a closed-loop feedback system. The circular piston section is linked to the rectangular test section by a $1 \mathrm{~m}$ long change of section. For the present study the test section was fitted with a false bottom elevated to $0.25 \mathrm{~m}$ above the tunnel floor, leaving $0.5 \mathrm{~m}$ water depth in the test section. The water depth was 


\author{
Change of section \\ (circular to rectangular)
}

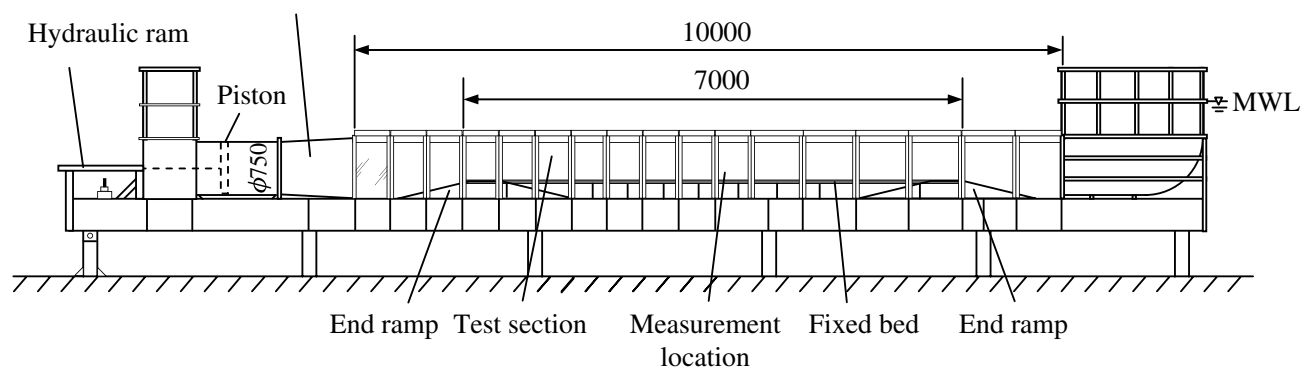

FIgURE 1. The Aberdeen oscillatory flow tunnel (dimensions in millimetres).

significantly larger than the thickness of the bottom boundary layer which for the present experiments was at most $0.05 \mathrm{~m}$. The thickness of the sidewall and roof boundary layers were not measured but are expected to be significantly smaller owing to the smooth surfaces. At both ends ramps were fitted with 1:4 slopes from the tunnel floor to the horizontal false bottom, allowing the test bed to be placed with a total length of $7 \mathrm{~m}$. Owing to the cross-sectional contraction from piston cylinder to test section, flows could be generated in the test section with orbital excursion amplitudes of $1.5 \mathrm{~m}$ for oscillatory flow periods greater than $5 \mathrm{~s}$.

The false floor construction consisted of a stainless steel support frame that was permanently bolted through the tunnel floor into the supporting main I-beams of the tunnel. The bed was made up of three $300 \mathrm{~mm}$ wide, $25 \mathrm{~mm}$ thick marine plywood panels bolted to the support frame at $500 \mathrm{~mm}$ intervals along the sidewalls and $1000 \mathrm{~mm}$ intervals along the tunnel centreline. The bed surface preparation began by brush-applying several coats of marine varnish onto the plywood panels, which were left to dry for several minutes to allow surface irregularities to settle out. For the experiments with sand roughness, a layer of the sand was spread over the wet varnished surface and tamped down, surplus sand was tipped off and the panels were left flat until completely dry. The gravel-rough bed was prepared in a similar way, but required extra varnish to secure individual grains to the plywood base. Before fitting the plywood panels the area of bed illuminated by the laser during PIV measurements was given a thin coat of matt black paint to reduce laser light reflection. The varnish layer did not affect the roughness as the grains lie on top of it and the black paint cover was sufficiently thin to have negligible effect on the bed roughness at the measurement location. Two small-diameter holes were made in the test bed at $500 \mathrm{~mm}$ either side of the PIV measurement location and fitted with tubes to enable injection of seeding particles in the tunnel test section prior to an experiment.

\title{
2.2. PIV measurements
}

The flow field was measured in the centre of the test section across the longitudinal midline of the tunnel using PIV. The flow was illuminated using a New Wave Research Solo III, double-pulsed Nd-Yag laser (532 nm wavelength). The laser beam was spread and focused into a light sheet by a series of spherical and cylindrical lenses and introduced to the tunnel flow through a $300 \mathrm{~mm}$ long, $25 \mathrm{~mm}$ wide glass slot cut centrally in the tunnel lid. In order to avoid disturbance of the laser sheet by bubbles crossing the sheet during an experiment, air bubbles that had formed under the tunnel lids were removed using vents built into the lids before commencing each 
experiment. For all experiments the water in the tunnel was seeded with silver-coated glass microspheres with a mean diameter of $10 \mu \mathrm{m}$ and specific gravity marginally greater than 1. A FlowSense 2 M $1600 \times 1200$ pixels CCD camera with a Nikon AF Micro Nikkor fixed focal length lens fitted with a $532 \mathrm{~nm}$ narrowband green filter was used for image acquisition. The timing of the laser pulses and synchronization with the camera was controlled by a Dantec Dynamics 80N53 Timer Box. For all experiments the time between images in a pair was $1 \mathrm{~ms}$ and image pairs were collected at a rate of $15 \mathrm{~Hz}$.

For most experiments, measurements were made with the camera positioned at two distances from the glass sidewall. At the first position the camera's field of view captured approximately the bottom $100 \mathrm{~mm}$ of the flow; for the second position the camera was positioned closer to the glass sidewall measuring approximately the bottom $50 \mathrm{~mm}$ of the flow, thereby increasing velocity resolution. Depending on the oscillatory flow period, either 4500 or 6000 image pairs were acquired during each experiment. The bed location was ascertained directly from the PIV images. The $z=0$ datum was taken to be the top of the uppermost grain crest in the measurement window.

\subsubsection{PIV data processing}

Image analysis was carried out using Dantec Dynamics' Dynamic Studio software. For each experiment the mean images of the even and odd frames were calculated from the image pair dataset. The mean images contain anything that stays stagnant in the image dataset, such as image background and light reflections from the bed. From every even and odd frame in the image dataset the respective mean image was subtracted, resulting in a background-extracted image dataset used for further processing. Vector processing was done using adaptive (or multi-pass) crosscorrelation, with three iterations and 50\% overlap: in the first iteration an interrogation area of $128 \times 128$ pixels was applied, reduced to $64 \times 64$ pixels in the second iteration and $32 \times 32$ pixels in the final interrogation. The physical size of the final interrogation areas was $2.5 \mathrm{~mm} \times 2.5 \mathrm{~mm}$ (camera position 1) or $1.1 \mathrm{~mm} \times 1.1 \mathrm{~mm}$ (camera position 2) for the sand-rough-bed experiments and $3.0 \mathrm{~mm} \times 3.0 \mathrm{~mm}$ or $1.4 \mathrm{~mm} \times 1.4 \mathrm{~mm}$ for the gravel-rough-bed experiments. Displacement information of the previous iteration was used as offset for the interrogation area in the next iteration following Westerweel, Dabiri \& Gharib (1997). PIV correlation peak displacement was assumed to be accurate to within 1/10th of a pixel (Westergaard et al. 2003) meaning that velocities were resolvable to within $7.9 \mathrm{~mm} \mathrm{~s}^{-1}$ (position 1) and $3.5 \mathrm{~mm} \mathrm{~s}^{-1}$ (position 2) for the sand-bed experiments and $9.5 \mathrm{~mm} \mathrm{~s}^{-1}$ and $4.3 \mathrm{~mm} \mathrm{~s}^{-1}$ for the gravel-bed experiments.

Erroneous flow vectors tended to occur in small clusters when seeding density was locally too low or a passing stray air bubble interrupted the light sheet. Erroneous vectors were detected using three different validation methods: (i) range validation, whereby vectors exceeding a prescribed velocity magnitude are rejected; (ii) signal-tonoise ratio validation of the correlation peak, whereby the highest and second highest correlation peaks within the interrogation area are compared and the vector is accepted if the ratio of the peaks is greater than a predefined value, taken to be 1.2 for the present experiments (Keane \& Adrian 1992); (iii) local-median validation, whereby a vector is rejected if it deviates by more than a prescribed amount from its surrounding neighbours (Westerweel 1994). The latter method was applied in the horizontal and time domain. Rejected vectors, generally between $1 \%$ and $2 \%$ of the vectors in the vector field, were replaced by their local median. 
Phase-averaged velocities were calculated by averaging over approximately 50 flow cycles and spatially averaging over several adjacent $x$-positions located around the centre of the PIV image:

$$
\langle\hat{\psi}\rangle(z, t)=\frac{1}{M} \sum_{m=1}^{M} \frac{1}{N} \sum_{n=1}^{N} \psi\left(x_{m}, z, t+(n-1) T\right), \quad 0 \leqslant t<T,
$$

where $\psi$ represents the velocity component ( $u$ or $w$ ), $N$ the number of flow cycles and $M$ the total number of $x$-locations used for spatially averaging $(M=45$ for the gravelrough bed and $M=70$ for the sand-rough bed). Spatially averaged root-mean-square (r.m.s.) turbulent fluctuations were calculated using

$$
\left\langle\left(\widehat{\psi^{\prime 2}}\right)^{1 / 2}\right\rangle(z, t)=\frac{1}{M} \sum_{m=1}^{M}\left[\frac{1}{N} \sum_{n=1}^{N} \psi\left(x_{m}, z, t+(n-1) T-\langle\hat{\psi}\rangle\right)^{2}\right]^{1 / 2}, \quad 0 \leqslant t<T,
$$

and spatially-averaged Reynolds stresses using

$$
\begin{aligned}
& -\rho\left\langle\widehat{u^{\prime} w^{\prime}}\right\rangle(z, t)=-\frac{\rho}{M} \sum_{m=1}^{M} \frac{1}{N} \sum_{n=1}^{N} u^{\prime}\left(x_{m}, z, t+(n-1) T\right) w^{\prime}\left(x_{m}, t+(n-1) T\right), \\
& 0 \leqslant t<T \text {, }
\end{aligned}
$$

where $\rho$ is the water density. Owing to the horizontal uniformity of the flow and the large interrogation area compared with the roughness size, spatially-averaging effectively serves as a way to increase the number of flow cycles for averaging. It should be noted that in (2.2), instead of subtracting spatially averaged, phase-averaged velocity $\langle\hat{\psi}\rangle(z, t)$, subtracting the local phase-averaged velocity $\hat{\psi}(x, z, t)$ does not lead to any difference in the r.m.s. fluctuations, except locally in the region very close to the bed $(z \leqslant 2 \mathrm{~mm})$ where the flow is directly affected by the presence of the roughness elements. Throughout the remainder of the paper, unless otherwise indicated, we simplify the notation by using $\psi(z, t)$ for $\langle\hat{\psi}\rangle(z, t)$ and $\psi_{r m s}(z, t)$ for $\left\langle\left(\widehat{\psi^{\prime 2}}\right)^{1 / 2}\right\rangle(z, t)$.

\subsection{LDA measurements}

The PIV measurements were supported by LDA point velocity measurements at a few locations above the bed. The much smaller measurement volume and the higher sampling frequency of the LDA measurements enables checking of the PIV results. A one-component backscatter LDA, powered by a Uniphase $20 \mathrm{~mW}$ He-Ne laser, was used to make separate $u$ and $w$ point velocity measurements at several elevations above the bed. The laser unit was fitted with a beam expander which resulted in a cylindrical measurement volume of $95 \mu \mathrm{m}$ in diameter and $800 \mu \mathrm{m}$ in length. Signal processing was achieved using a Dantec 57N10 burst spectrum analyser (BSA). Horizontal velocities were measured at six elevations spaced logarithmically above the grain crest $(z=0)$, at $z=1.3,5.0,14.8,29,61$ and $117 \mathrm{~mm}$ for the sand-rough-bed experiments and $z=1.6,5.4,14.8,29,61$ and $112 \mathrm{~mm}$ for the gravel-rough-bed experiments. Vertical velocities were measured by rotating the laser optics through $90^{\circ}$. However, the angles of the laser beams meant that measurement points within approximately $14 \mathrm{~mm}$ of the bed could not be accessed by the lowest laser beam, therefore vertical velocities were only measured at the four upper elevations. At each elevation data were collected for 75 flow cycles. The velocity measurements 
were phase-locked with a once-per-flow-cycle pulse sent to the BSA from the PC controlling the piston motion and each flow cycle was divided into equi-sized phase intervals for averaging. Phase-averaged velocities and r.m.s turbulent fluctuations over the duration of the 75 flow cycles were obtained by incorporating the residence time weighting to remove velocity bias, as described by Bucchave, George \& Lumley (1979). For the present experiments bin sizes of $2^{\circ}$ and $1.5^{\circ}$ were used for flows with $5 \mathrm{~s}$ and $7 \mathrm{~s}$ period respectively. Phase-averaged results for bins with less than 50 data points were rejected.

\section{Experimental conditions}

The experiments involved a total of six flows over two fixed beds with different roughness. One bed consisted of coarse sand with a median diameter $d_{50}=0.44 \mathrm{~mm}$; the second consisted of gravel with grain diameter between 5.0 and $6.3 \mathrm{~mm}$. The piston control software was programmed to generate a free-stream velocity (i.e. above the bottom boundary layer) in the tunnel test section given by

$$
u_{0}(t)=\alpha u_{0 \max } \sum_{i=1}^{6} \frac{(2 \beta-1)^{i-1} \sin i \omega t}{i}
$$

where $\omega=2 \pi / T, T$ is the flow period, $u_{0 \max }$ is the maximum free-stream velocity and the value of the multiplier $\alpha$ is chosen such that the product of $\alpha$ and the maximum value of the summation is equal to unity (the maximum of $u_{0}(t)$ is then equal to $\left.u_{0 \max }\right) ; \beta$ is the acceleration-skewness parameter defined as

$$
\beta=\frac{\dot{u}_{0 \max }}{\dot{u}_{0 \max }-\dot{u}_{0 \min }}
$$

where $\dot{u}_{0}$ is the free-stream flow acceleration. The test conditions are presented in table 1 . The six flows comprised two flow periods of $5 \mathrm{~s}$ and $7 \mathrm{~s}$, each with three degrees of acceleration skewness: near sinusoidal flow $(\beta \approx 0.5)$ and two accelerationskewed flows with $\beta \approx 0.6$ and $\beta \approx 0.75$. These are realistic $\beta$ values for coastal zones, since typical values are in the range $0.43 \leqslant \beta \leqslant 0.85$ as can be inferred from a large collection of field measurements by Elfrink et al. (2006). The $u_{0 \max }$ and $u_{0 \text { min }}$ values in table 1 are the measured maximum and minimum free-stream velocities. For most conditions $u_{0 \max }$ and $u_{0 \min }$ were not exactly equal in magnitude owing to a slight departure from pure acceleration-skewed flow in the test section, which means that the flows contained a small degree of velocity skewness $R\left(=u_{0 \max } /\left(u_{0 \min }-u_{0 \min }\right)\right)$. Example phase-averaged PIV-measured time series of free-stream horizontal velocity - experiments S507012g and S757012g - are shown in figure 2. The acceleration time series shown were obtained from the reconstituted first six harmonics of the measured velocity and the values of $\beta$ were obtained from the resulting maximum positive and negative accelerations given by (3.2). Orbital amplitude has been determined from the measured free-stream velocity time series using $a=\sqrt{2} u_{0} \mathrm{rms} / \omega$, where $u_{0 \mathrm{rms}}$ is the root-mean-square of the free-stream velocity. The roughness length, $k_{s}$, was found from applying the log-law to the measured velocity profiles (see $\S 6$ ). The values quoted in table 1 are overall $k_{s}$ values for the two different rough beds. Reynolds number, $R e$, is given by $R e=u_{0 \text { max }} a / v$ where $v$ is the kinematic viscosity of water, equal to $1.12 \times 10^{-6} \mathrm{~m}^{2} \mathrm{~s}^{-1}$ for the present experiments. Note that measurements with the camera at the second position closest to the glass sidewall were only conducted for eight experiments. 

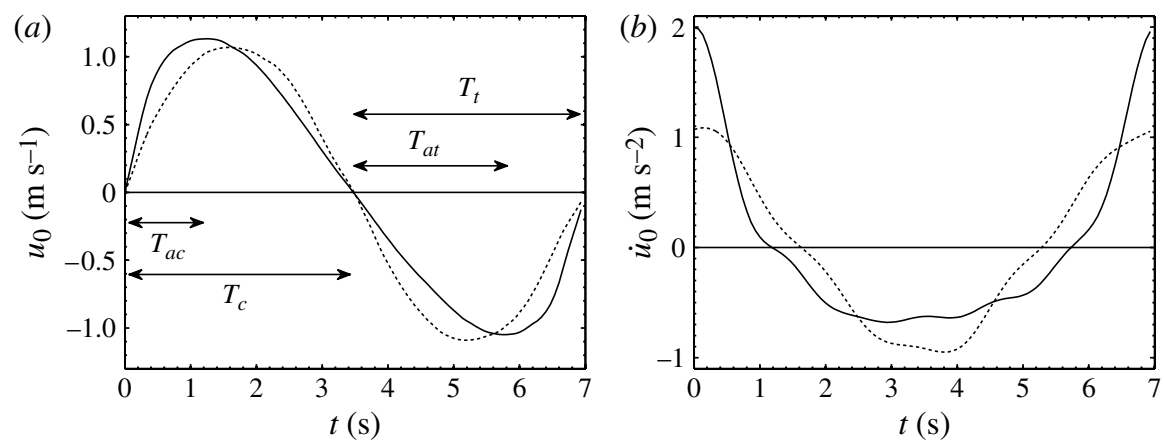

FIgURE 2. Time series of $(a)$ free-stream velocity and $(b)$ free-stream acceleration for the gravel-rough bed, $T=7 \mathrm{~s}$ and $\beta=0.53$ (S507012g, dashed) and $\beta=0.75$ (S757012g, solid). $T_{c}$ and $T_{t}$ indicate the positive ('crest') and negative ('trough') half-cycle duration, respectively. $T_{a c}$ and $T_{a t}$ indicate the duration of positive flow acceleration and duration of negative flow acceleration for the $\beta=0.75$ condition.

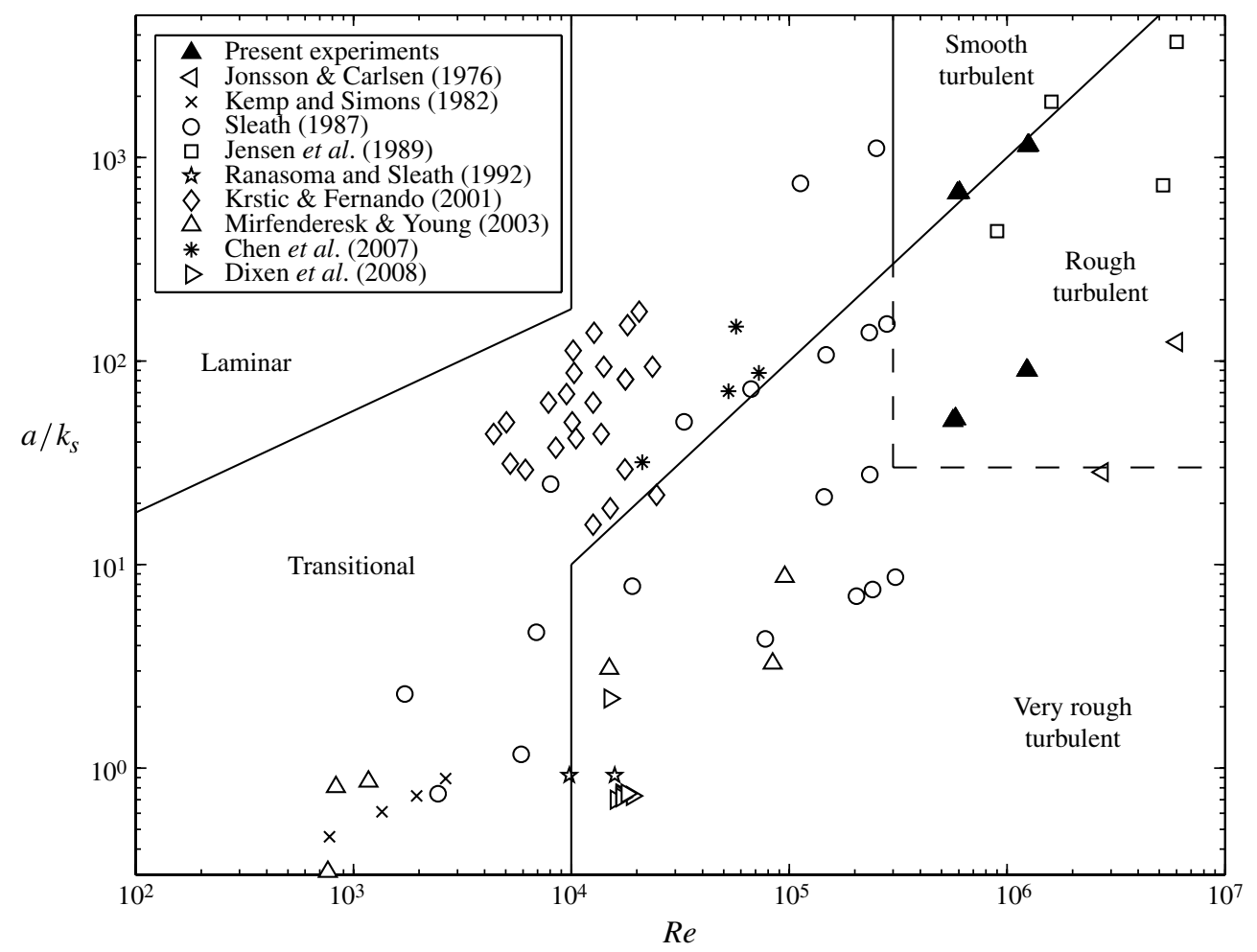

FIGURE 3. Delineation of flow regimes for oscillatory flow illustrating the present experiments and previous studies.

Figure 3 positions the present experiments in $R e-a / k_{s}$ space along with the flow tunnel conditions of Jonsson \& Carlsen (1976), Sleath (1987) and Jensen et al. (1989). The solid lines indicate Jonsson's (1980) recommended criteria for the boundaries between the laminar, transitional and turbulent flow regimes. The dashed 

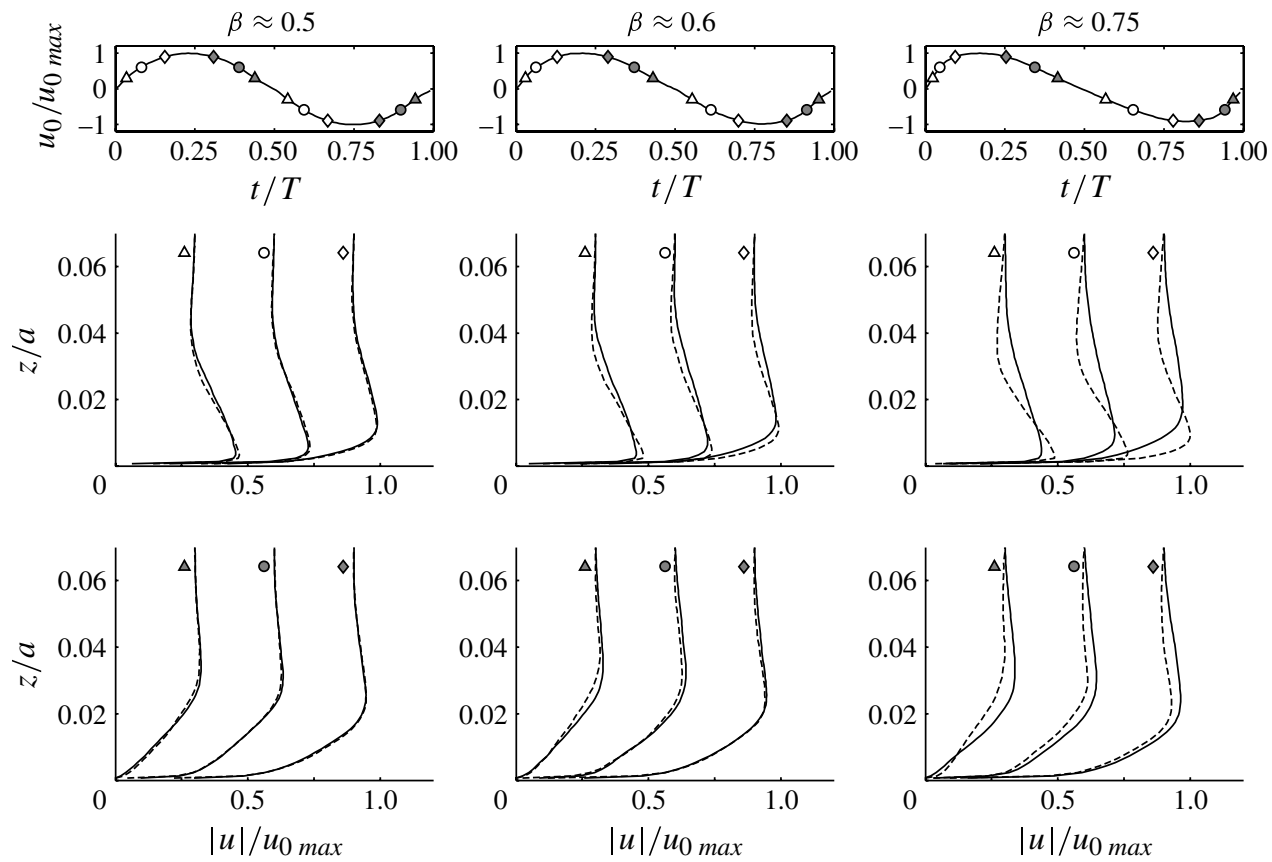

FIGURE 4. Velocity profiles corresponding to accelerating phases (open symbols) and decelerating phases (closed symbols) of the flow for $T=7 \mathrm{~s}$, above the sand-rough bed and for three different degrees of acceleration skewness $\beta$ : dashed line, positive flow profiles; solid line, negative flow profiles. Pairs of velocity profiles correspond to $\left|u_{0}\right| / u_{0 \text { max }}=0.3$ (triangle), 0.6 (circle) and 0.9 (diamond).

line indicates the transition from the rough turbulent to the very rough turbulent regime in which momentum transfer is mainly due to vortex shedding from individual roughness elements (Davies \& Villaret 1997). For example, seabeds covered with ripples (e.g. Ranasoma \& Sleath 1992) or rock armour (e.g Dixen et al. 2008) belong to this regime, so also do most of Sleath's (1987) experiments with gravel-rough and pebble-rough beds. Considering that for typical field conditions over flat sand beds $R e>10^{5}$ and $a / k_{s}>12.5$ (Nielsen 1992), it is clear from figure 3 that the present experiments add substantially to the existing experimental data in this range. Furthermore, the Jonsson \& Carlsen (1976), Sleath (1987) and Jensen et al. (1989) tunnel experiments were confined to sinusoidal flows, while the present experiments cover both sinusoidal and acceleration-skewed flows (note that each marker for the present experiments represents three flow conditions corresponding to three values of $\beta$ ). Conditions corresponding to the wave flume experiments of Kemp \& Simons (1982) and Mirfenderesk \& Young (2003), to the oscillating plate experiments of Krstic \& Fernando (2001), and to the oscillatory open channel experiments of Chen et al. (2007) are also shown, but these are all confined to low $R e$ values.

\section{Phase-averaged velocities}

\subsection{Velocity profiles}

The effect of acceleration skewness on the boundary layer velocity profile is seen clearly in figure 4 . Each of the parts of figure 4 contains three pairs of velocity 
profiles. The profiles in each pair correspond to two phases within the flow cycle when the free-stream velocity magnitude is the same (but different in direction), but with the acceleration or deceleration being different depending upon $\beta$. Consider first the results for accelerating flow (the upper profiles). For $\beta \approx 0.5$, i.e. near-sinusoidal flow with zero acceleration skewness, profiles with the same magnitude of free-stream velocity are nearly identical: the profiles are the same whether the flow is positive or negative because the accelerations are the same. For increasing acceleration skewness, increasing differences can be seen between the high-acceleration, positive flow profiles and the corresponding low-acceleration, negative flow profiles: peak near-bed velocities and near-bed velocity gradients are greater for the high-acceleration positive flow, implying higher bed shear stress during positive flow compared to the negative flow (analysed further in §6). This behaviour is analogous to that of a steady flow velocity profile subject to a favourable pressure gradient: the greater the favourable pressure gradient, the greater the near-bed velocity gradient. In our case, higher (lower) flow acceleration corresponds to greater (lower) favourable pressure gradient (since in the free stream $\partial u_{0} / \partial t=-(1 / \rho) \partial p / \partial x$ assuming horizontal uniform flow), which leads to higher (lower) velocity gradient. Consider now the velocity profiles corresponding to decelerating flow (the lower panels in figure 4). Again, profiles for corresponding positive and negative flow are almost identical for the near-sinusoidal flow condition. Differences can be seen between the positive and negative flow profiles as $\beta$ increases, but the differences here are much smaller compared with the differences observed for the accelerating flow. These differences can similarly be explained, at least qualitatively, by analogy with steady flow velocity profiles subject to adverse pressure gradients.

The detailed structure of the boundary layer velocity profile may be seen by harmonically analysing the flow at each elevation and plotting the $z$-profiles of the harmonic amplitudes and phases. This is done in figure 5 for the $5 \mathrm{~s}$ and $7 \mathrm{~s}$ flows over the sand-rough and gravel-rough beds. The behaviour of the harmonics is remarkably consistent for the two flow periods and two bed types. The firstharmonic amplitude shows the expected overshoot and phase lead of the near-bed flow relative to the free stream, the phase lead becoming larger for larger relative roughness (i.e. lower $a / k_{s}$ ). The second-harmonic amplitude increases with increasing acceleration skewness, as intended according to (3.1), with $\left|u_{2}\right|_{0} \approx 0$ for $\beta \approx 0.5$ and $\left|u_{2}\right|_{0}$ increasing with increasing $\beta$. The absence of any significant change in $\left|u_{2}\right|$ with height suggests that this term is not strongly associated with nonlinear behaviour in the bottom boundary layer turbulence. It appears that the second-harmonic amplitude for the $T=5 \mathrm{~s}$ conditions is relatively larger than for the $T=7 \mathrm{~s}$ conditions. The reason for this difference is unclear since it is not imposed following (3.1) and it is not seen in the case of the third-harmonic amplitudes. In contrast to the reasonably constant second harmonic with depth, the third harmonic grows in relative importance towards the bed, and it does so to a relatively greater extent for $\beta \approx 0.5$ than for $\beta \approx 0.6$ and 0.75 . At the same time the oscillating nature with height of the phase of the third harmonic is also far more pronounced for $\beta \approx 0.5$ than for $\beta \approx 0.6$ and 0.75 , highlighting very strong nonlinear interaction in the boundary layer for $\beta \approx 0.5$ and still pronounced, but weaker, interaction for $\beta \approx 0.6$ and 0.75 .

\subsection{Phase lead}

A measure of the phase difference, $\phi$, between the free stream and the near-bed flow used by previous researchers (Jonsson \& Carlsen 1976; Jensen et al. 1989; Dixen et al. 2008) is the phase difference between the occurrence of maximum free-stream velocity 

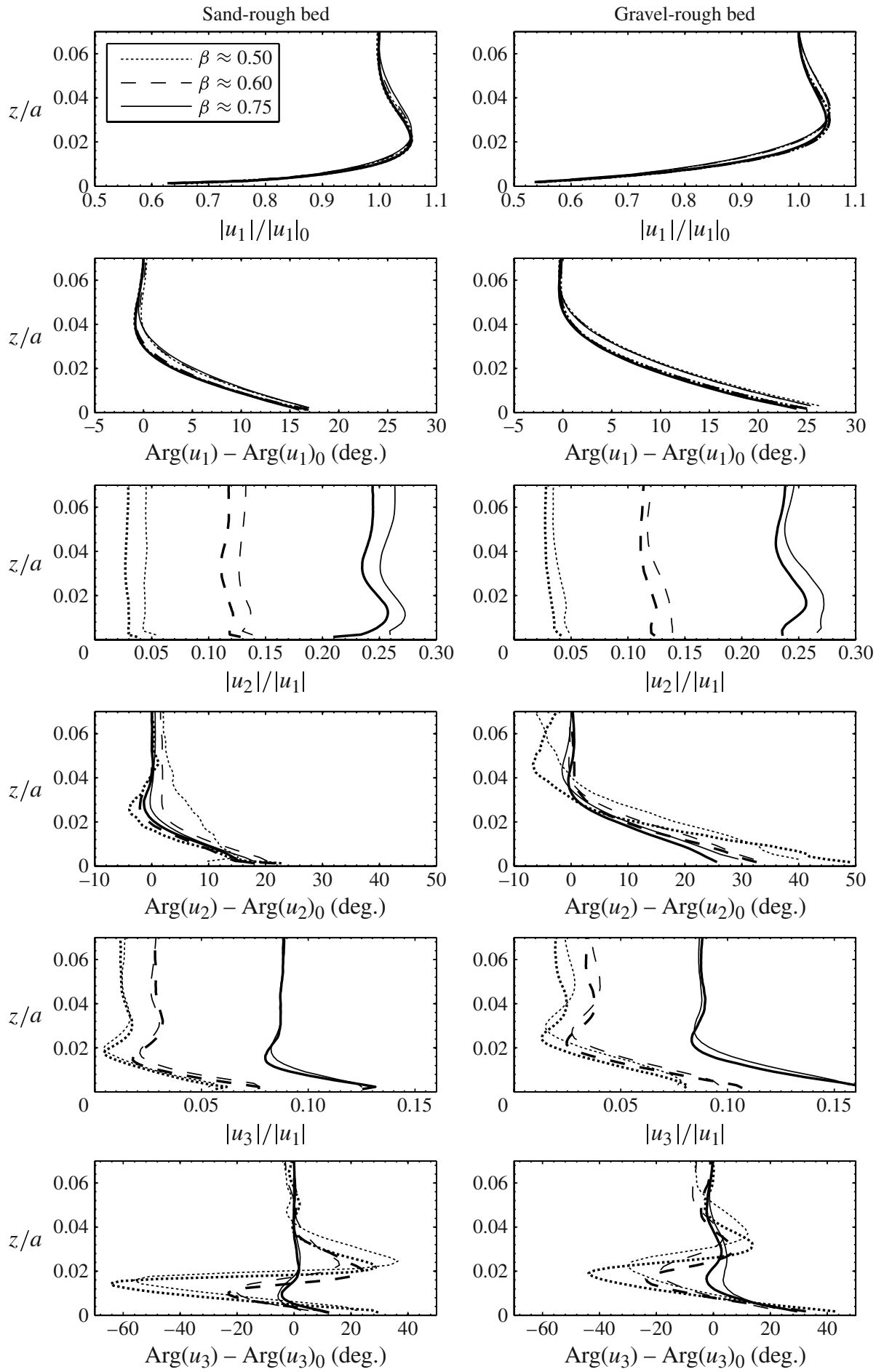

FIGURE 5. Amplitude and phase plots for the first three fundamental harmonics; thinner lines $T=5 \mathrm{~s}$; thicker lines $T=7 \mathrm{~s}$. The first-harmonic amplitude $\left|u_{1}\right|$ is normalized by its free-stream value $\left|u_{1}\right|_{0}$, higher-harmonic amplitudes $\left|u_{2}\right|$ and $\left|u_{3}\right|$ are normalized by the firstharmonic amplitude at each elevation. Harmonic phases are shown relative to the phase of the free-stream flow. 


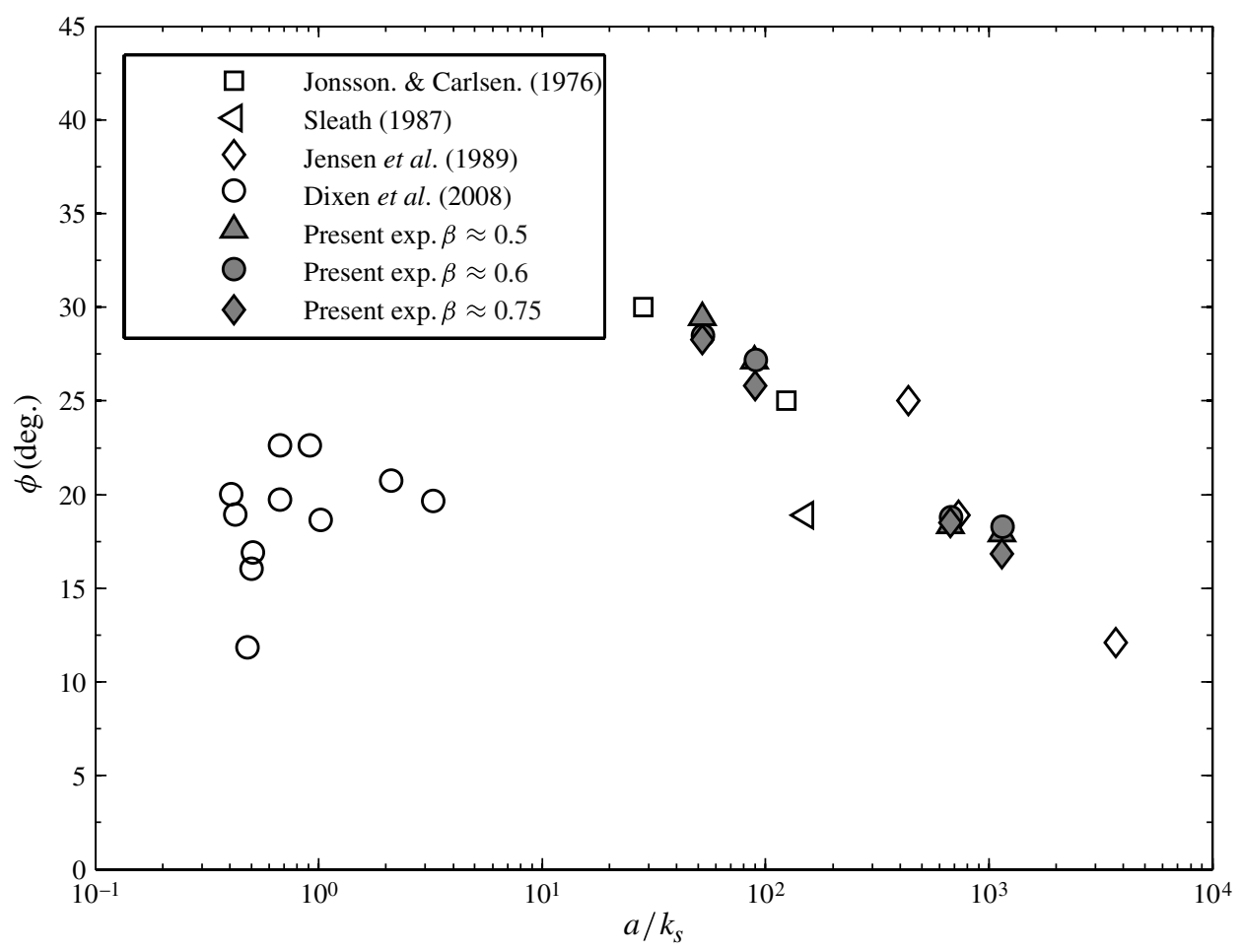

FIGURE 6. First-harmonic phase lead as a function of $a / k_{s}$ compared to existing studies.

and maximum friction (shear) velocity, which for smooth, laminar simple harmonic flow has a value of $45^{\circ}$. To compare phase-lead values from the present experiments with those from previous flow tunnel experiments with simple harmonic flow, $\phi$ is defined as the phase of the first harmonic extrapolated to the bed. The comparison is presented in figure 6 , which shows that the values of $\phi$ thus obtained from the present experiments are very consistent with existing results. The new experiments confirm the existence of a logarithmic relationship between $\phi$ and $a / k_{s}$ in figure 6 for large $a / k_{s}$ (i.e. for rough turbulent flow). The results of Dixen et al. (2008) illustrate that for very low $a / k_{s}$ the logarithmic relationship does not apply.

With regard to the present experiments, an interesting aspect for consideration is the phase shift that occurs in individual half-cycles of the flow. Figure $7(a)$ shows the positive-half-cycle phase lead, $\phi_{\text {pos }}(z)$, for three values of $\beta$ for the $T=7 \mathrm{~s}$ gravel bed condition. Here $\phi_{\text {pos }}(z)$ is defined as the phase difference between the instant of positive-to-negative flow reversal in the free stream and that at elevation $z$. Figure $7(b)$ shows the negative-half-cycle phase lead, $\phi_{\text {neg }}(z)$, which corresponds to the phase difference of the instant of negative-to-positive flow reversal in the free stream and that at elevation $z$. Figure $7(a)$ shows that, when looking downwards from free-stream level, the phase shift becomes apparent from $z / a \approx 0.05$ and the phase lead becomes increasingly larger as the bed is approached. It appears, moreover, that $\phi_{\text {pos }}(z)$ is consistently larger for larger $\beta$. In contrast, figure $7(b)$ shows that $\phi_{\text {neg }}$ becomes apparent at a lower level, and $\phi_{\text {neg }}$ is consistently smaller for increasing $\beta$. For example for $\beta \approx 0.75$, near the bed $\phi_{\text {pos }} \approx 32^{\circ}$ while $\phi_{\text {neg }}$ is only $15^{\circ}$. For $\beta \approx 0.5$ there appears to be no significant differences between $\phi_{\text {pos }}$ and $\phi_{\text {neg }}$, as expected. The 

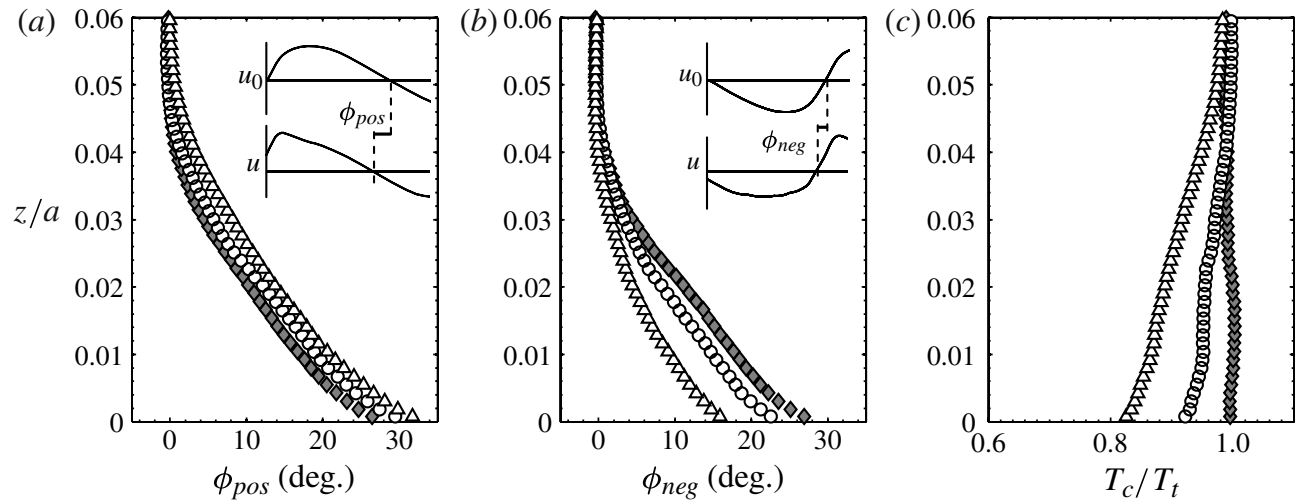

Figure 7. (a) Positive-half-cycle phase lead; $(b)$ negative-half-cycle phase lead; $(c)$ ratio of crest and trough duration. $\beta \approx 0.5$ (diamonds), $\beta \approx 0.6$ (circles), $\beta \approx 0.75$ (triangles). $T=7 \mathrm{~s}$, gravel-rough bed.

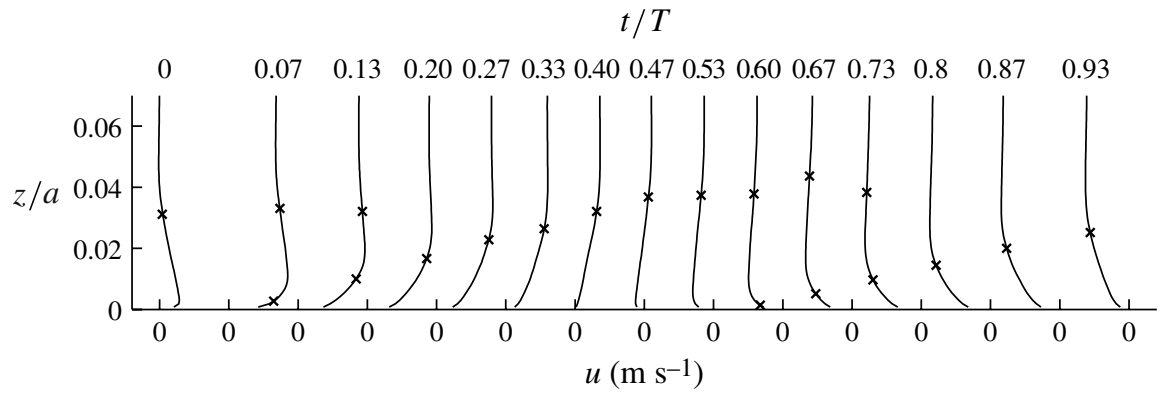

FIGURE 8. Boundary layer thickness $(\times)$ superimposed on velocity profiles (S757012g).

consequence of this asymmetry in phase lead between both half-cycles is that the duration of the positive half-cycle, $T_{c}$, is shorter than the duration of the negative halfcycle, $T_{t}$. This effect is further illustrated in figure $7(c)$, which shows the ratio $T_{c} / T_{t}$ for the same conditions. It can be seen that for the acceleration-skewed flows $T_{c} / T_{t}$ decreases as the bed is approached and that this difference becomes more pronounced for larger $\beta$.

\subsection{Boundary layer thickness}

A common definition of boundary layer thickness, $\delta$, for oscillatory flow is the distance from the bed to the elevation at which the velocity defect (here the magnitude of the difference between the free-stream velocity and the velocity within the boundary layer) equals $1 \%$ of $u_{0 \max }$ at the moment of maximum free-stream velocity. The intra-wave boundary layer thickness, $\delta(t)$, can similarly be obtained based on the instantaneous velocity defect $u_{d}(z, t)=u_{0}(t)-u(z, t)$. Because $u_{d}(z)$ has the nature of a damped oscillation propagating upwards, two values of $\delta(t)$ can be obtained for many phases of the flow cycle: one corresponding to the first elevation from the bed upwards where $u_{d}(z)$ meets the threshold value and one corresponding to the first elevation from the free-stream level downwards where $u_{d}(z)$ meets the threshold value. This is illustrated in figure 8 for experiment S757012g. Owing to experimental scatter in the defect velocity measurements, a threshold for $u_{d}$ corresponding to $5 \%$ of $u_{0 \max }$ 

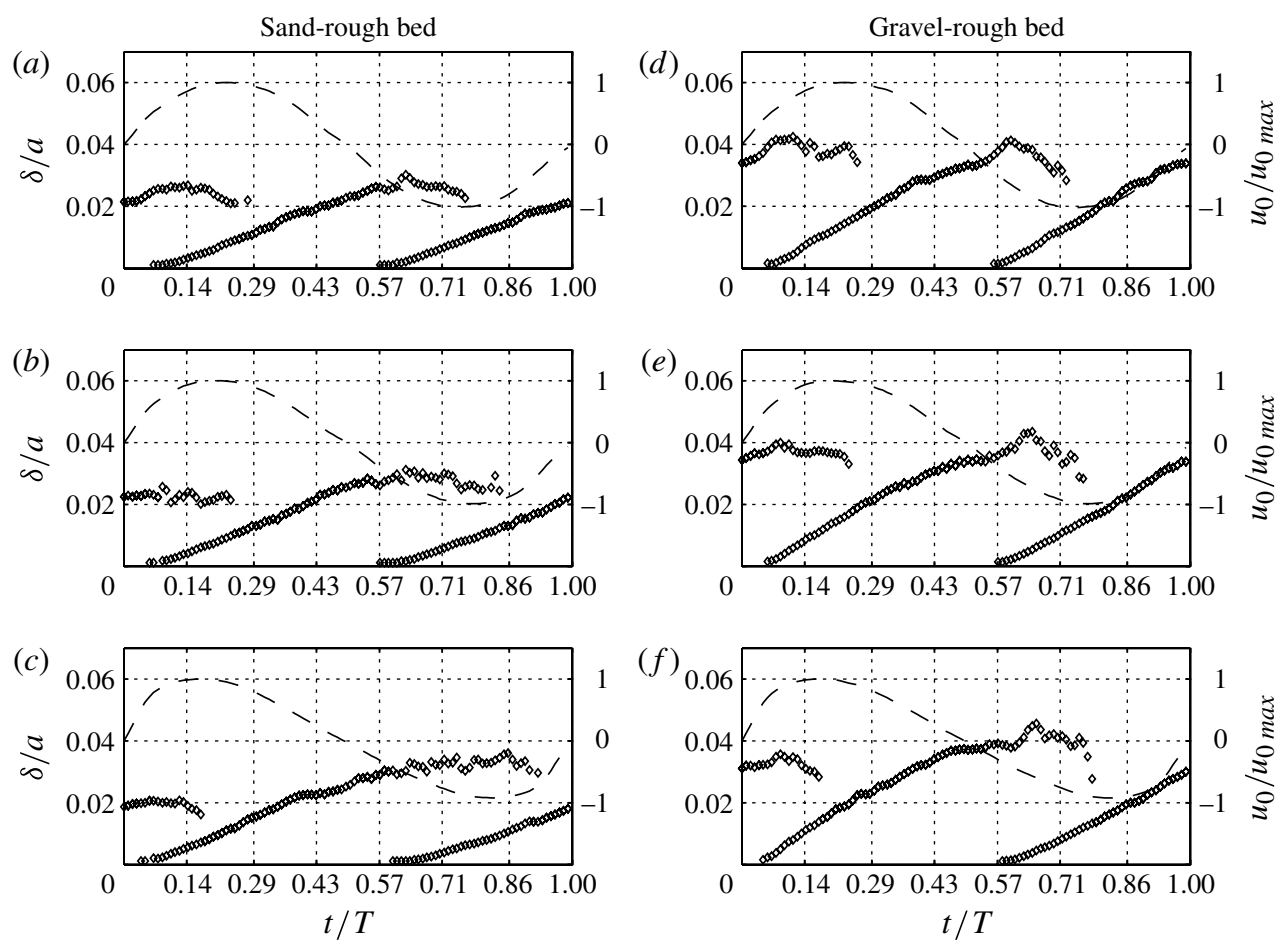

FIgURE 9. Time-dependent total boundary layer thickness: $(a, d) \beta \approx 0.54,(b, e) \beta=0.62$, $(c, f) \beta=0.75$. The dashed line represents the normalized free-stream velocity. $T=7 \mathrm{~s}$, $u_{0 \max } \approx 1.1 \mathrm{~m} \mathrm{~s}^{-1}$.

in determining $\delta(t)$ is applied (rather than 1\%), similar to Sleath (1987). It is seen that the two measures of the boundary layer thickness coexist during the accelerating stages of the flow (i.e. following $t / T=0$ and 0.5 ) because of the velocity overshoot near the bed. The time series of boundary layer thickness for the $7 \mathrm{~s}$ period flow over the two bed types are presented in figure 9; the two measures of $\delta(t)$ occur at most phases of the flow cycle as seen in these time series. It can also be seen how the 'inner' boundary layer thickness grows as the flow velocity increases following the times of free-stream flow reversal, as expected. Note that the measurements do not show $\delta$ starting to increase exactly at times of free-stream flow reversal because the lowest measurements are somewhat above $z=0$ and because of the relatively high threshold value of $5 \%$ of $u_{0 \max }$ (as opposed to $1 \%$ ). In all cases, the boundary layer grows from the time of flow reversal and continues to grow both during flow acceleration to maximum free-stream velocity and also during flow deceleration towards flow reversal, after which the new near-bed bottom boundary layer starts near the bed and the upper one levels off and persists approximately until maximum freestream flow in the following half-cycle. The (inner) boundary layer growth depends on the flow period such that, for a given free-stream velocity amplitude, the larger the flow period, the greater the orbital excursion, and the thicker the boundary layer becomes. For the acceleration-skewed flows of interest here $(\beta=0.62,0.75)$, the accelerating phase of the positive half-cycle, from $t / T=0$ to peak positive velocity, is of significantly shorter duration than its negative counterpart from $t / T=0.5$ to peak negative velocity. The shorter time limits the boundary layer growth, as seen in 


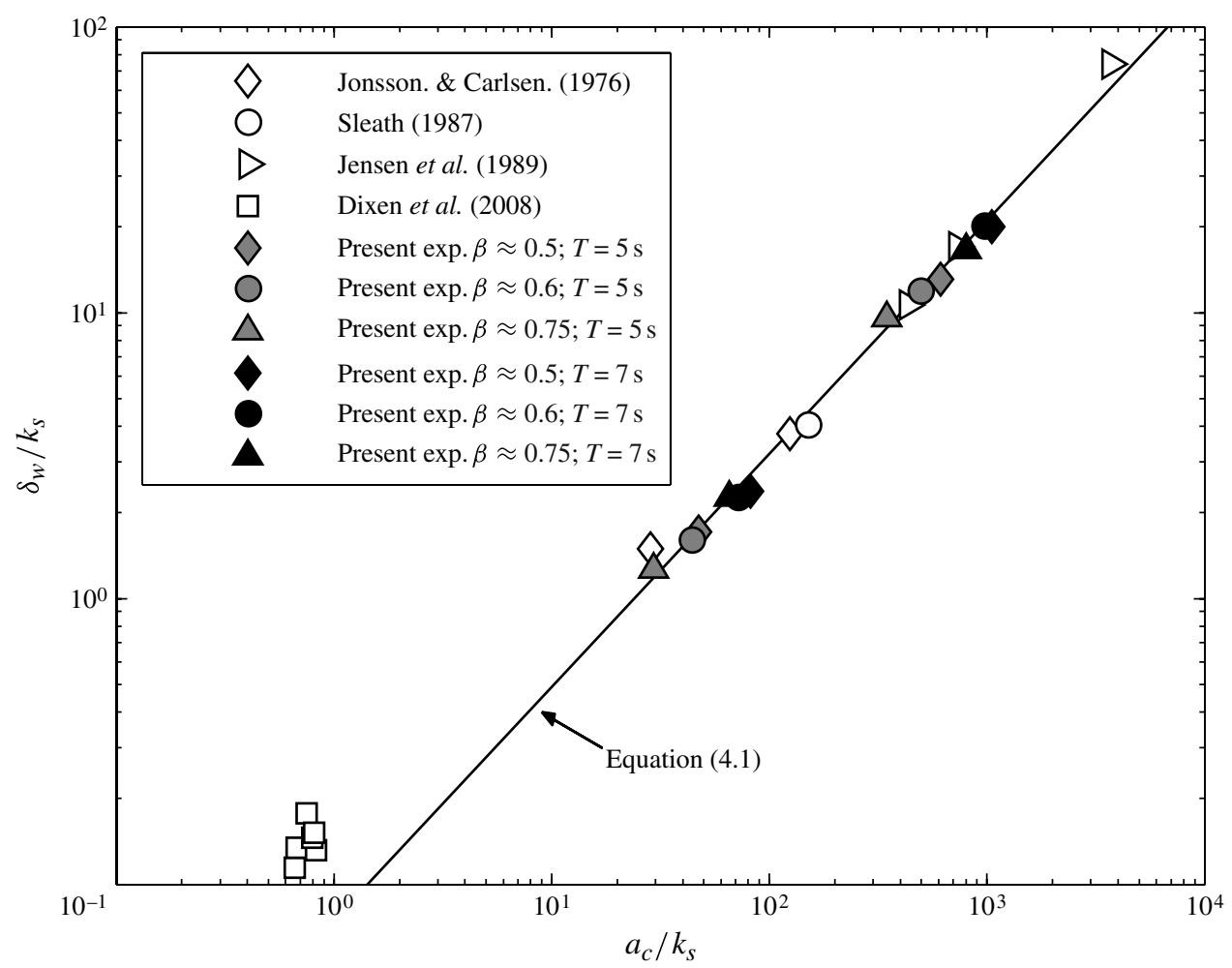

FIGURE 10 . Boundary layer thickness versus $a_{c} / k_{s}$. Here $\delta$ is defined as the elevation of maximum overshoot following Jensen et al. (1989).

figure 9 and as also seen previously in the velocity profiles (figure 4). As described by Nielsen (1992), and as will be shown in $\S 6$, this leads to a distinct asymmetry in bed shear stress between the two half-cycles. Figure 9 shows furthermore that the boundary layer develops more rapidly when acceleration increases; compare for example the positive-half-cycle-generated $\delta$ with the negative-half-cycle-generated $\delta$ in figure $9(f)$. The rate of boundary layer growth $\mathrm{d} \delta / \mathrm{d} t$ for the former is much greater compared to the latter. As observed by Sumer et al. (1993) for their converging-diverging flow and further illustrated in $\S 5$, this asymmetry in time evolution of $\delta$ is closely related to asymmetry in turbulence development each flow half-cycle.

The effect of bed roughness on the boundary layer thickness is clear in figure 9 from the much greater boundary layer thickness of the gravel-rough bed compared with the sand-rough bed. The effect of $a / k_{s}$ is further illustrated in figure 10 , which shows the boundary layer thickness (normalized by $k_{s}$ ) plotted as a function of the relative roughness, $a / k_{s}$. To facilitate comparison with existing data, $\delta_{w}$ is defined as the distance from the bed to the point of maximum velocity overshoot when $u_{0}(t)=u_{0 \text { max }}$, following Jensen et al. (1989). For increasing acceleration skewness the moment of maximum free-stream velocity occurs sooner in the oscillatory flow cycle, which as already mentioned has a limiting effect on the excursion between $t=0$ and the moment of free-stream flow. This effect is accounted for in the results in figure 10 by using an equivalent sinusoidal orbital amplitude, $a_{c}$, for $a$, where $a_{c}=a 2 T_{a c} / T_{c}$ following Silva, Temperville \& Seabra-Santos (2006). For flow without acceleration 

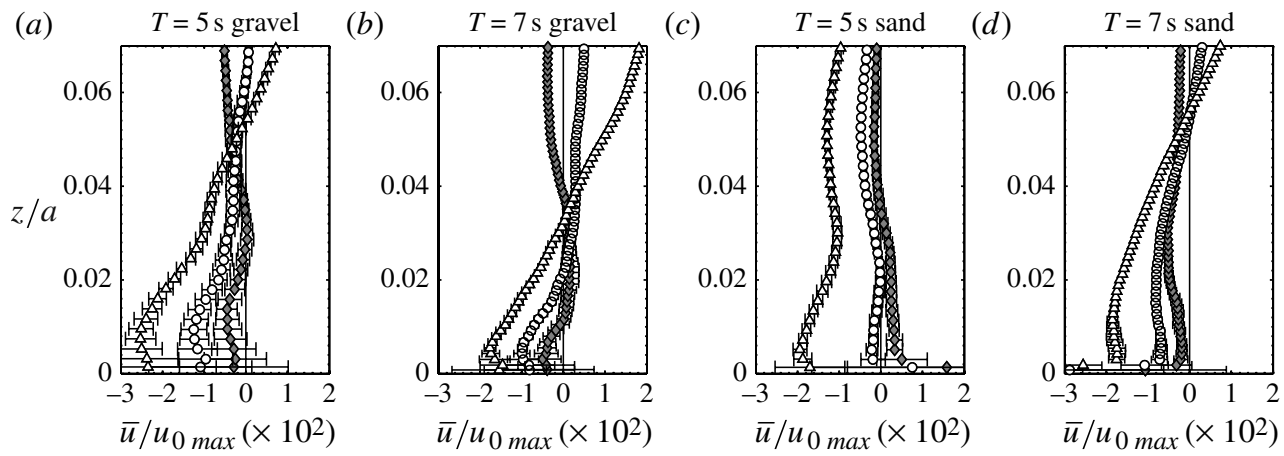

FIGURE 11. Time-averaged residual flow $\bar{u}$ for all conditions. Error bars indicate the \pm 1 standard deviation variation across all $x$-positions within the two-dimensional PIV window.

skewness, $a_{c}=a$ (which is the case for all results from previous studies contained in figure 10). The results from the present experiments are seen to agree extremely well with those from previous experiments, confirming the increasing trend in $\delta_{w} / k_{s}$ with increasing $a / k_{s}$. Based on a general relationship where $\delta_{w} / k_{s}=m\left(a / k_{s}\right)^{n}$ (see e.g. Jonsson \& Carlsen 1976; Sleath 1987; Fredsøe \& Deigaard 1992), the results in figure 10 (excluding those of Dixen et al. (2008) which show no clear relationship with $a / k_{s}$ ) suggest the following relationship between $\delta_{w} / k_{s}$ and $a_{c} / k_{s}$ :

$$
\frac{\delta_{w}}{k_{s}}=0.075\left(\frac{a_{c}}{k_{s}}\right)^{0.82},
$$

which closely resembles Fredsøe \& Deigaard (1992) who proposed $m=0.09$ and $n=0.82$.

\subsection{Time-averaged flow}

Nonlinear intra-wave processes in the turbulent oscillatory boundary layer give rise to a time-averaged flow in the boundary layer. The residual velocity profile is

$$
\bar{u}(z)=\frac{1}{T} \int_{0}^{T} u(z, t) \mathrm{d} t .
$$

For velocity-skewed flows the residual is against the direction of the largest velocity and has been measured in mobile-bed flow tunnel experiments by Ribberink \& Al-Salem (1995) and O'Donoghue \& Wright (2004). The mechanism giving rise to the residual velocity is the asymmetry in turbulence intensity between the two flow half-cycles, which leads to the generation of a time-averaged, height-dependent stress within the boundary layer (Trowbridge \& Madsen 1984; Davies \& Li 1997; Holmedal \& Myrhaug 2006). Figure 11 presents the residual velocity profiles for all of the present 12 experiments. For the near-sinusoidal flows $(\beta \approx 0.5)$, the residual is close to zero, as expected (although not exactly zero because the flow is not exactly sinusoidal). The near-bed residual is negative and increases in magnitude with increasing $\beta$, indicating increasing asymmetry in turbulence between the two half-cycles. This negative residual not only occurs near the bed but also at the tunnel's sidewalls and lid. Hence, for reasons of mass continuity in the AOFT, the negative residual near the bed must be balanced by net positive flow in the centre of the 
tunnel, which is seen at higher elevations in figure 11. The maximum of $\bar{u}$ in the negative direction is approximately $2 \%$ of the maximum free-stream velocity for highest $\beta$. This agrees reasonably well with Fuhrman et al. (2009) who, by solving the incompressible Reynolds-averaged Navier-Stokes equations combined with a $k-\omega$ turbulence model, showed $\bar{u}$ to be slightly less than $2 \%$ of $u_{0 \max }$ for rough turbulent flow with $\beta=0.67$. Comparing the present measurements with those of Ribberink \& Al-Salem (1995) for velocity-skewed flow $(R=0.66)$ over mobile beds in which $\bar{u} / u_{0 \text { max }} \approx 0.03$, the magnitude of residual driven by acceleration skewness seems to be smaller than that driven by velocity skewness.

Furthermore, for given $\beta$, there appears to be no significant difference in the maximum (normalized) residual for different flow period and bed roughness. This is consistent with Holmedal \& Myrhaug (2006) who, using a $k-\varepsilon$ model, showed there was no significant effect of $a / k_{s}$ on the near-bed residual for velocity-skewed flow. They indicated that although the time-averaged stress will increase with increasing roughness, leading to a larger negative mean flow (in the case of an infinite tunnel), the finite length of the tunnel will lead to a stronger return flow with increasing roughness, which consequently does not lead to significant changes in the residual velocity profile. The increase in magnitude of $\bar{u}$ with increasing acceleration skewness may be analogues to the results of Sumer et al. (1993), who found the magnitude of $\bar{u}$ to increase with increasing convective acceleration $(u(\partial u / \partial x))$ in their convergent-divergent tunnel experiments. However, in Sumer et al.'s study $\bar{u}$ was in the same direction as the mean bed shear stress whereas in our study it is directed against the mean positive shear stress. This reason for this difference in direction has, for example, been discussed by Fuhrman et al. (2009).

\subsection{Vertical velocities}

Although in theory the flow in the tunnel test section should be purely horizontal, the phase- and spatial-averaged vertical velocities were non-zero, albeit very small compared with the horizontal velocities. Figure 12(a) presents example time series of phase- and spatial-averaged vertical velocities at several elevations above the gravelrough bed. At the two lowest elevations shown in figure 12(a) the vertical velocities are strongly periodic around a negative mean flow, with frequency equal to twice the frequency of the horizontal oscillatory flow, whereas at higher elevations the periodicity and mean offset have largely disappeared. The close proximity to the bed suggests that the former patterns are generated by the flow negotiating individual gravel particles of the bed. In fact similar observations were made by Sleath (1987) who attributed the near-bed periodicity of the vertical flow to 'local irregularities' of his $1.63 \mathrm{~mm}$ sand bed. However, in Sleath's (1987) case the periodicity appeared in the ensemble-averaged vertical velocity measured at a single $x$-location, while the vertical velocity time series presented in figure 12(a) are spatially averaged over 45 adjacent locations within the PIV measurement window. In principle, the spatial- and time-averaged vertical velocity will tend to zero as the bed is approached, since upward localized jet formation linked to flow around individual gravel particles should be compensated by simultaneous downward motions of fluid between the jets. However, it is seen from the measurements at the bottom two elevations in figure $12(a)$, and in more detail from the time-averaged vertical velocity profiles in figure $12(b)$, that measured $\bar{w}$ is actually negative below approximately $z=10 \mathrm{~mm}$. The most likely explanation for this disparity between measurements and expectation is that, at least for the gravel-bed conditions, the averaging window (here equivalent to five gravel particles) is too small to reliably capture the mean vertical velocity, 

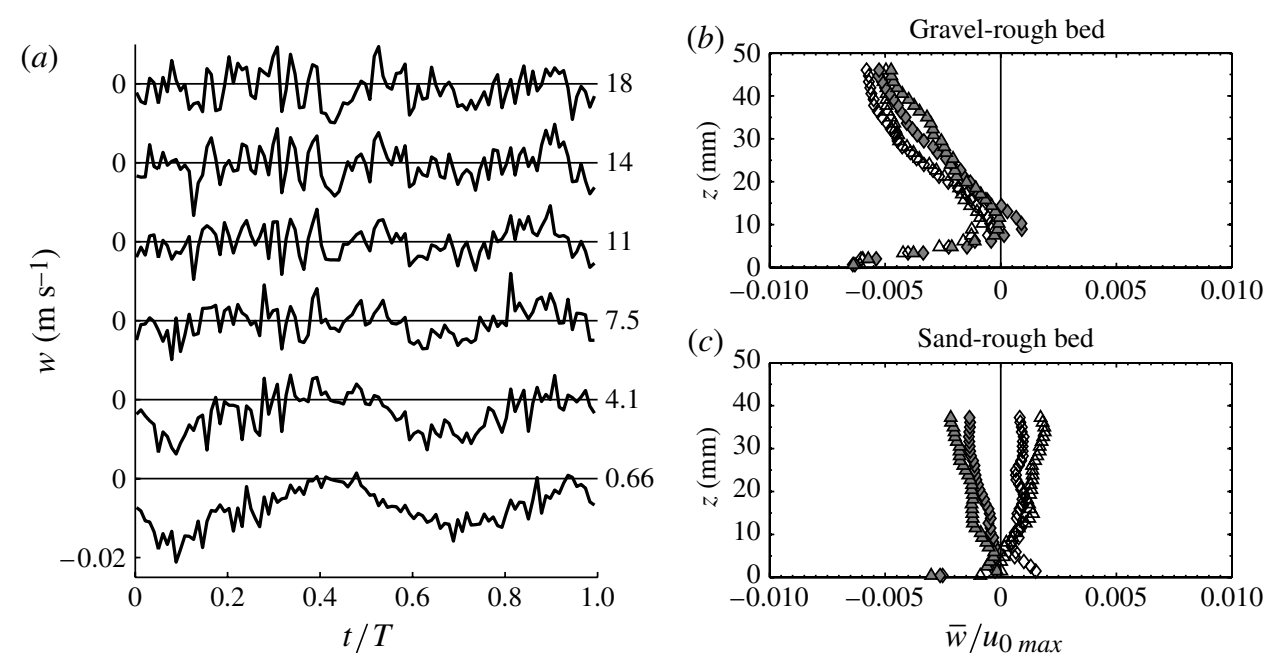

FIGURE 12. (a) Example time series of phase- and spatial-averaged vertical velocities at selected elevations above the bed (indicated on the right in $\mathrm{mm}$; note that consecutive $y$ axes are offset by $0.02 \mathrm{~m} \mathrm{~s}^{-1}$ ), $\beta=0.75, T=7 \mathrm{~s}$, gravel-rough bed. Time-averaged vertical velocity profiles: $(b)$ gravel-rough bed; $(c)$ sand-rough bed. Open symbols: $T=5 \mathrm{~s}$ conditions; closed symbols: $T=7 \mathrm{~s}$ conditions.

introducing bias into the near-bed spatial-averaged vertical velocities below $z \approx 10 \mathrm{~mm}$. This explanation is supported by the fact that the time-averaged vertical flow near the bed is generally close to zero in the case of the sand-rough bed, as seen in figure $12(c)$. In the sand-rough case, the averaging window contains approximately 70 grains and is likely to be sufficiently wide to properly capture the time-averaged flow.

There is no significant effect of $\beta$ on the $\bar{w}$ profiles and an effect of the flow period (or $a$ ) is only seen in the coarse-sand conditions; the profiles for the gravel-rough bed are remarkably consistent (note that the vertical axis is not normalized here). An explanation for the existence of a non-zero $\bar{w}$ at away from the near-bed region is the presence of secondary currents in the tunnel driven by differences in wall friction between the bed and tunnel sidewalls. Such frictional differences can induce an upward drift along the tunnel sidewalls and hence a downward drift along the centreline. The frictional differences would be greater when the bed is rougher, thus creating a larger downward drift, consistent with the results in figure $12(b, c)$. The implications of these small vertical velocities are further discussed in $\S 6$.

\section{Turbulence}

\subsection{Turbulent fluctuations}

Figure 13(a) presents PIV-measured vertical profiles of horizontal turbulence intensity, $u_{r m s}^{\prime}$, corresponding to 10 phases of the flow period for the $5 \mathrm{~s}$ flow with highest acceleration skewness $(\beta=0.75)$ over the sand-rough bed. The top row of profiles corresponds to the positive-flow half-cycle and the bottom row to the negative-flow half-cycle. The LDA-measured turbulence intensities are also shown and remarkably good agreement is seen between the PIV and LDA results. Note that LDA measurements are available for fewer elevations at times around flow reversal because of the phase-averaging method. The profiles illustrate that turbulence builds up near the bed during each flow half-cycle and then diffuses away from the bed through 

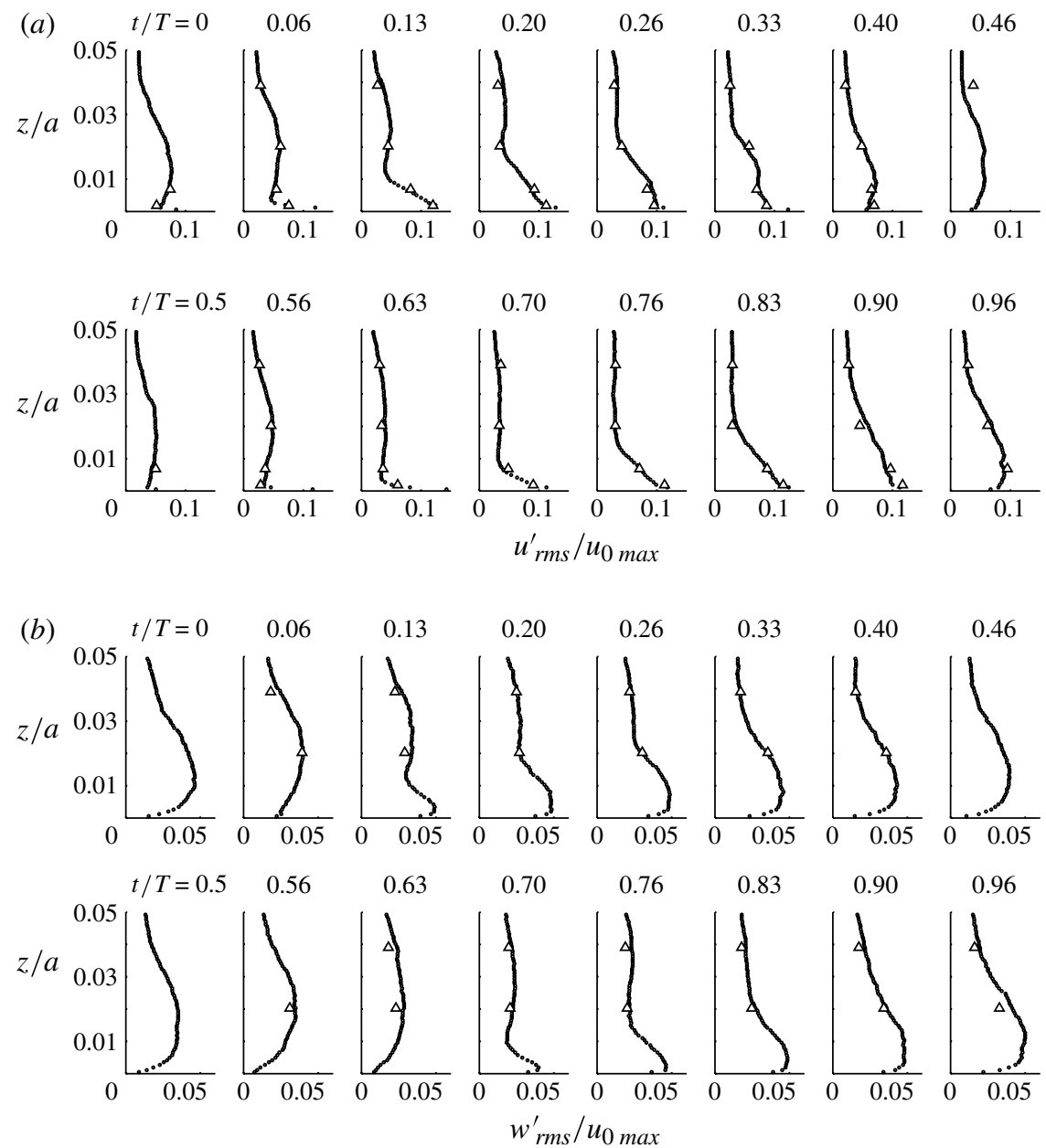

FIGURE 13. (a) Profiles of horizontal turbulence intensity $u_{r m s}^{\prime}$, and (b) vertical turbulence intensity $w_{r m s}^{\prime}$ : dots, PIV measurements; triangles, LDA measurements. Experiment S755010c.

the boundary layer. During the positive half-cycle maximum $u_{r m s}^{\prime}$ occurs at the bed close to the phase of maximum free-stream flow $(t / T=0.13)$, i.e. at the end of flow acceleration, which is in agreement with the previous measurements of Sleath (1987) and Jensen et al. (1989) for sinusoidal flows. At about this time the vertical gradient in intensity is high near the bed, for $z / a<0.015$. As the flow decelerates the turbulence intensity decreases, the peak in intensity propagates upwards and the high vertical gradient near the bed decays. Intensities from the positive half-cycle persist into the accelerating phase of the following negative half-cycle during which the highest $u_{r m s}^{\prime}$ again occurs at the bed, increasing and propagating higher into the flow with increasing flow velocity. However, turbulence build-up near the bed occurs again during later phases of this half-cycle because maximum free-stream velocity occurs relatively late (at $t / T=0.83$ ), due to the high degree of acceleration skewness. The negative-to-positive flow reversal at $t / T=0$ occurs relatively soon after maximum negative velocity with the result that there are higher intensities at this time compared with those at the positive-to-negative flow reversal at $t / T=0.5$. The behaviour seen 

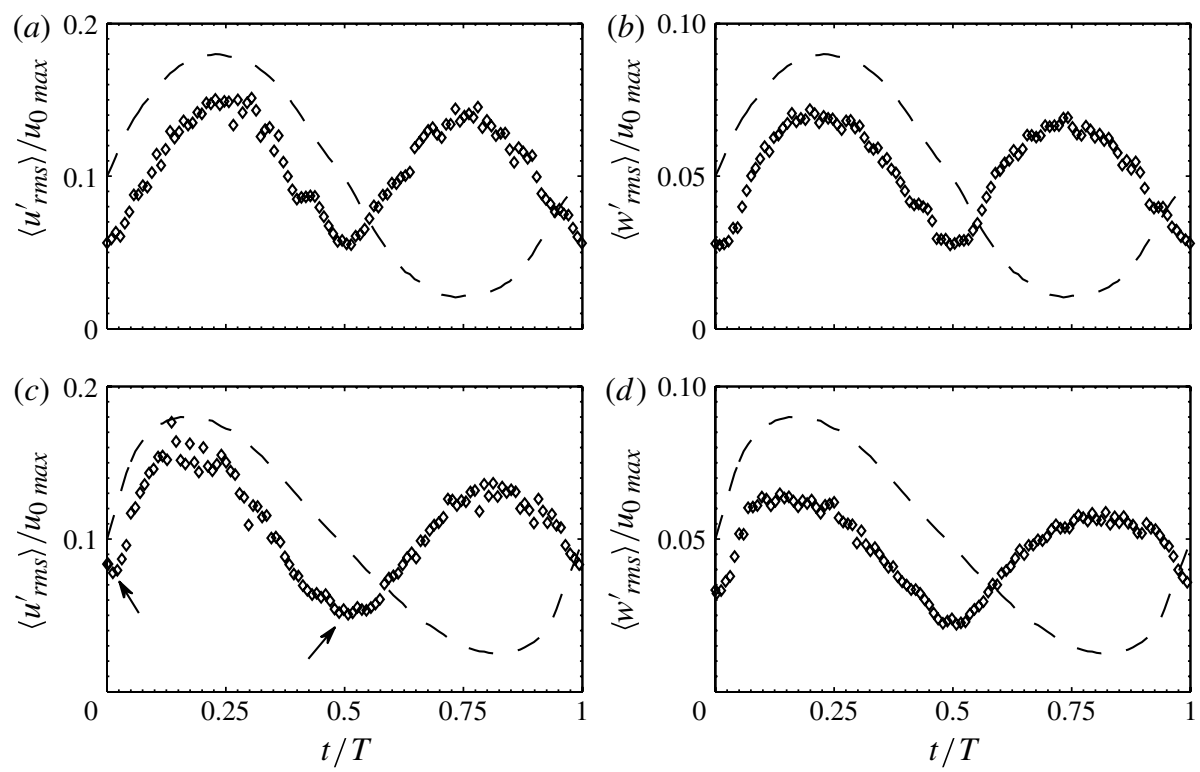

FIGURE 14. Time series of $u_{r m s}^{\prime}$ for $(a) \beta=0.53$ and $(c) \beta=0.75$, and corresponding $w_{r m s}^{\prime}$ times series for $(b) \beta=0.53$ and $(d) \beta=0.75$, at $z \approx 3 \mathrm{~mm}$, gravel-rough bed. The dashed line represents the free-stream flow cycle (not to scale).

in figure 13 echoes that described by Sumer et al. (1993) for converging-diverging flow, where turbulence from the diverging-flow half-cycle is seen to persist into the converging-flow half-cycle and vice-versa.

Profiles of vertical turbulence intensity, $w_{r m s}^{\prime}$, are presented for the same 10 phases in figure $13(b)$. As expected, $w_{r m s}^{\prime}$ is smaller than $u_{r m s}^{\prime}$, here smaller by about a factor two, in agreement with previous laboratory (e.g. Sleath 1987; Jensen et al. 1989; Sumer et al. 1993; Chen et al. 2007) and field observations (Soulsby 1983). The behaviour of the $w_{r m s}^{\prime}$ profiles through the flow cycle is similar to that seen for $u_{r m s}^{\prime}$, with turbulence being generated near the bed, peaking near the time of maximum flow velocity and diffusing upwards during the half-cycle. A noticeable difference between the $w_{r m s}^{\prime}$ and the $u_{r m s}^{\prime}$ profiles is that peak $u_{r m s}^{\prime}$ occurs at the bed while peak $w_{r m s}^{\prime}$ occurs at some small distance above the bed. This may be expected since the presence of the bed limits vertical water particle fluctuations. As for $u_{r m s}^{\prime}$, significantly higher turbulence intensities occur at the negative-to-positive flow reversal compared with the positive-to-negative flow reversal. The $u_{r m s}^{\prime}$ and $w_{r m s}^{\prime}$ behaviour seen in figure 13 is also seen in results from the other experiments (not shown here for brevity). The excellent agreement between PIV and LDA results was seen for all experiments, and turbulence intensities are greater for experiments with larger roughness as expected.

Figure 14 presents example PIV-derived time series of horizontal and vertical turbulence intensity for two experiments: near-sinusoidal flow (experiment S507012g) and acceleration-skewed flow (experiment S757012g), both over the gravel-rough bed measured at $z \approx 3 \mathrm{~mm}$. Much of the time-dependent behaviour in turbulence intensity described earlier is also seen in figure 14: increasing intensity during accelerating flow, peak intensity near maximum free-stream velocity and greater intensity at the negative-to-positive compared to the positive-to-negative flow reversal. In addition, the rate of increase in turbulence intensity during accelerating positive and accelerating 

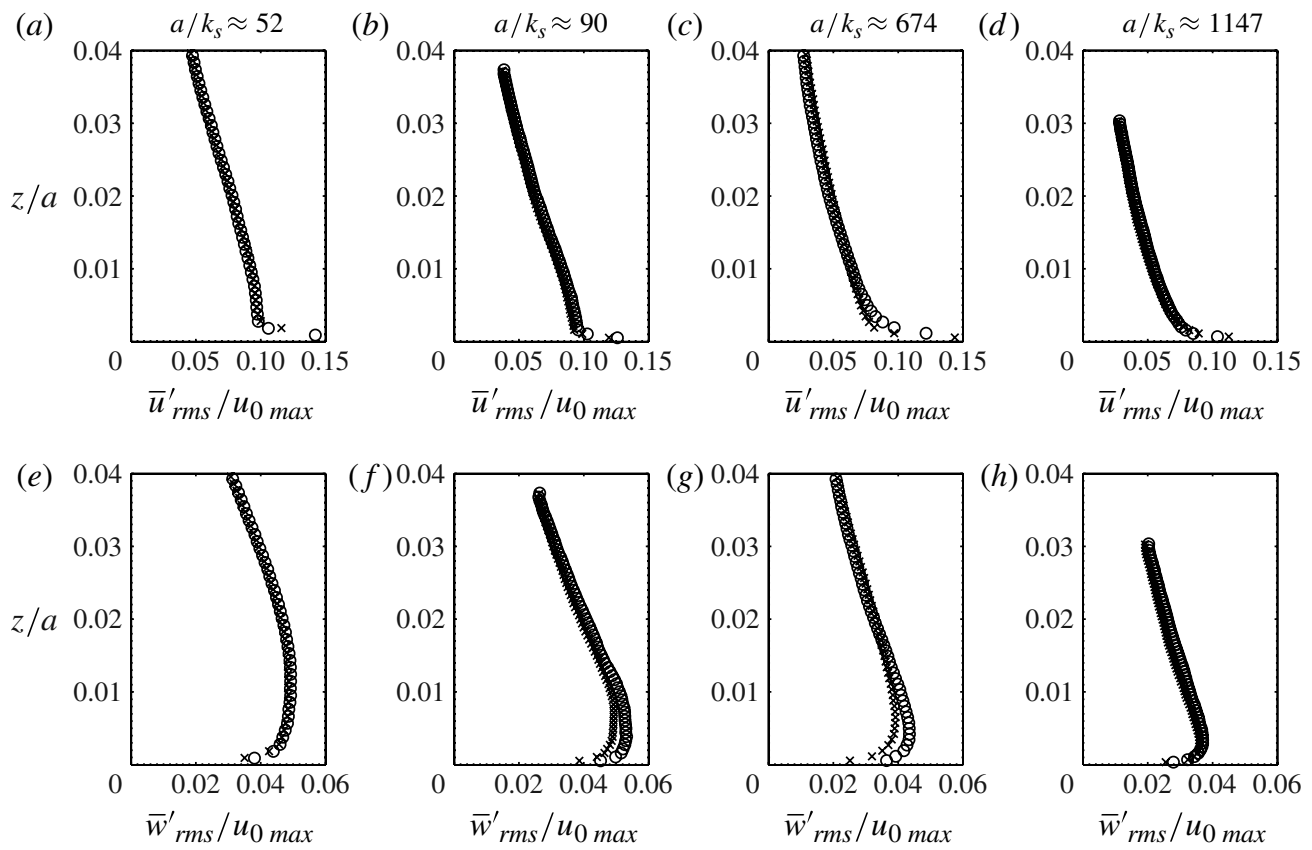

FIGURE 15. Time-averaged (averaged over flow cycle) $u_{r m s}^{\prime}(a-d)$ and $w_{r m s}^{\prime}(e-h)$ profiles; $\beta \approx 0.5(\circ), \beta \approx 0.75(\times)$.

negative flow in each time series closely resembles the free-stream velocity. The asymmetry in the rate of increase of turbulence intensity caused by acceleration skewness is clear: the more-or-less symmetrical distribution in the case of sinusoidal flow contrasts with the dissimilar slopes in the case of the acceleration-skewed flow, showing a much greater rate of increase in intensity and larger peak intensity during the high-acceleration positive flow compared with the low-acceleration negative flow. Consequently, as indicated by the arrows in figure 14(c), the levels of turbulence intensity at both flow reversals are dissimilar for the acceleration-skewed flow.

Vertical profiles of time-averaged $u_{r m s}^{\prime}$ and $w_{r m s}^{\prime}$ are presented in figure 15, with each sub-figure containing results for the two extreme $\beta$ values (nominally 0.5 and 0.75 ) for the same $T, u_{0 \max }$ and bed roughness. The effect of $\beta$ on the profiles of $\bar{u}_{r m s}^{\prime}$ is minimal, despite $\beta$ having significant effect on intra-wave $u_{r m s}^{\prime}$ (figure 14). Timeaveraged $u_{r m s}^{\prime}$ increases with increasing proximity to the bed. In contrast to Sleath's (1987) corresponding results for sinusoidal flow, which show a peak in $\bar{u}_{r m s}^{\prime}$ very close to the bed and a decrease towards zero at the bed consistent with the no-slip condition, the present results show a high gradient of increasing $\bar{u}_{r m s}^{\prime}$ in the immediate proximity of the bed, which presumably is a result of local flow variations in this region due to the direct influence of the grains and variation related to the PIV interrogation areas partially overlapping the grains. Time-averaged $u_{r m s}^{\prime} / u_{0 \text { max }}$ is greater for the gravelrough bed $\left(a / k_{s} \approx 52,90\right)$ than for the sand-rough bed $\left(a / k_{s} \approx 674,1147\right)$, as expected. The effect of $\beta$ on $\bar{w}_{r m s}^{\prime}$ is also minimal, again despite $\beta$ having significant effect on intra-wave $w_{r m s}^{\prime}$. Also, $\bar{w}_{r m s}^{\prime}$ is a factor 2 less than $\bar{u}_{r m s}^{\prime}$, reflecting earlier observations from the intra-wave turbulence intensity profiles. The value of ${\overline{w^{\prime}}}_{r m s}$ increases with increasing proximity to the bed, attains a maximum and, in contrast to $\bar{u}_{r m s}^{\prime}$, then 

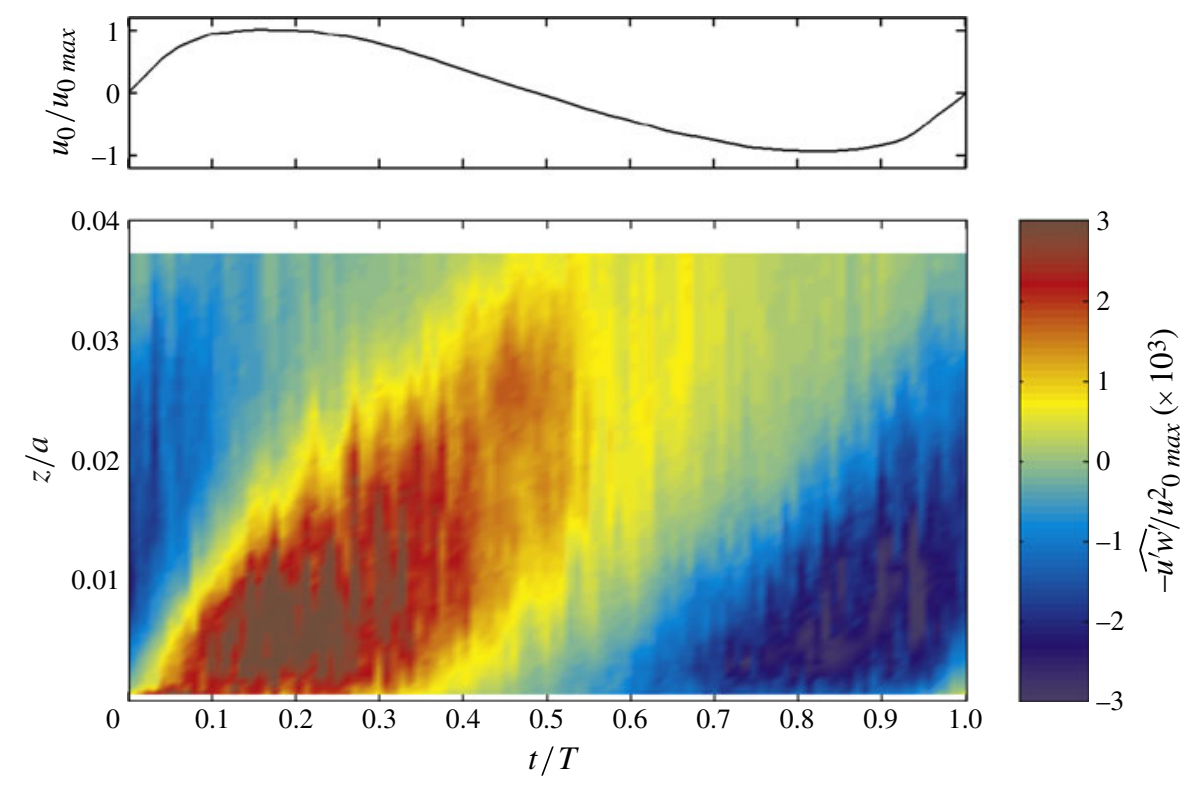

FIGURE 16. Contour plot showing Reynolds stress variation with height and phase.

Gravel-rough bed, $\beta=0.75, T=7 \mathrm{~s}$.

decreases very close to the bed. The elevation at which this maximum occurs increases with increasing roughness (decreasing $a / k_{s}$ ).

\subsection{Reynolds shear stress}

A contour plot of the Reynolds stress variation with height and phase is shown in figure 16. The figure echoes results already presented for turbulence intensity: $-\widehat{u^{\prime} w^{\prime}}$ increases near the bed as the flow accelerates during the positive half-cycle, decays as the free-stream flow decelerates, increases again as the flow accelerates during the negative half-cycle and decays again as the free-stream flow decelerates once again. Peak $-\widehat{u^{\prime} w^{\prime}}$ occurs first at the bed and increasingly later in time with increasing distance from the bed, reflecting the fact that the turbulence is predominantly bedgenerated and during the course of the cycle is redistributed in the vertical direction. The acceleration skewness means that largest $-\widehat{u^{\prime} w^{\prime}}$ occurs relatively early during the positive half-cycle, and since there is a relatively long time until the following flow reversal $(t / T \approx 0.5)$ it has mostly decayed by flow reversal. During the negative halfcycle on the other hand, $-\widehat{u^{\prime} w^{\prime}}$ remains small during the first stages $(t / T \approx 0.5-0.7)$, is largest at the end of the half-cycle and, because of the short time until flow reversal, persists much more into the following positive half-cycle. For mobile beds, this difference in timing of the (Reynolds) stresses between each half-cycle can have a significant effect on the net sand transport, especially for fine sands with relatively low settling velocity where the timing differences can augment so-called phase-lag effects (e.g. Dohmen-Janssen et al. 2002).

The similarities between the sharp contours in figure 16 and the behaviour of the time-dependent boundary layer thickness (figure 10) show how the turbulence development coincides with the boundary layer development. For this particular condition there also appears to be a strong asymmetry in the magnitude of the turbulent stress between the two half-cycles, with the magnitude of $-\widehat{u^{\prime} w^{\prime}}$ largest 

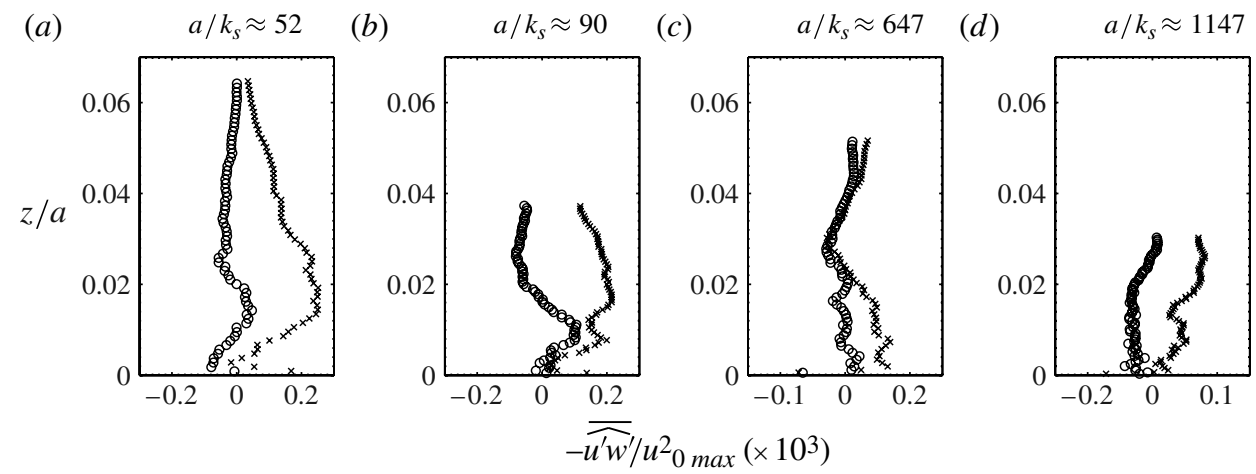

FIGURE 17. Time-averaged profiles of Reynolds shear stress; $\beta \approx 0.5(\circ), \beta \approx 0.75(\times)$.

during the positive half-cycle. It should be mentioned that such a distinct asymmetry between the two half-cycles did not occur for all experiments with acceleration-skewed flow, even though the asymmetry was clearly reflected in the periodic behaviour. However, the influence of $\beta$ on the Reynolds stress is seen in figure 17, which presents (normalized) time-averaged profiles of Reynolds stress. Despite some scatter close to the bed, figure 17 shows that the time-averaged Reynolds stress is generally largest and positive for $\beta \approx 0.75$.

\section{Bed shear stress}

The velocity measurements allow estimates of the intra-wave bed shear stress to be obtained in two ways: (i) via log-law fitting to the measured instantaneous velocity profiles; (ii) by application of the momentum equation for horizontally uniform oscillatory boundary layer flow to the measured velocities.

\subsection{Log-law}

For sinusoidal oscillatory flow over rough beds, Sleath (1987) and Jensen et al. (1989) both showed the existence of a logarithmic layer for large periods of the oscillatory flow cycle, where the velocity profile within the log layer is given by

$$
u(z)=\frac{u_{*}}{\kappa} \ln \left(\frac{z+d^{\prime}}{z_{0}}\right),
$$

with friction velocity $u_{*}=\sqrt{\tau / \rho}, d^{\prime}$ is the displacement thickness, von Kármán constant $\kappa=0.4$ and $z_{0}=k_{s} / 30$. The log profile is contained within the constantstress layer with upper bound given by $z+d^{\prime}=(0.2-0.3) \delta$, and lower bound away from any direct influence of the roughness elements on $u$, e.g. $z+d^{\prime} \geqslant 0.2 k_{s}$ (Grass 1971). Fitting (6.1) to measured instantaneous profiles, with $d^{\prime}, z_{0}$ and $u_{*}$ all varying to optimise the fit, leads to temporal variation in bottom roughness, which is not realistic since roughness should be constant if the boundary layer is fully developed and rough turbulent (e.g. Cox et al. 1996). Therefore, the representative values of $z_{0}$ and $d^{\prime}$ for each of the sand-rough and gravel-rough beds were established as follows: the log profile was fitted to the profile of the amplitude of the first harmonic, $u_{1}(z)$, for each experiment and the resulting $z_{0}$ and $d^{\prime}$ for the same bed type were averaged. For the gravel-rough bed this resulted in $d^{\prime}=0.29 d_{50}$ and $k_{s}=2.44 d_{50}$ (averaged over the six experiments, with standard deviation in $k_{s}$ of $0.11 d_{50}$ ). These results are in good 
Turbulent boundary layer in acceleration-skewed flow
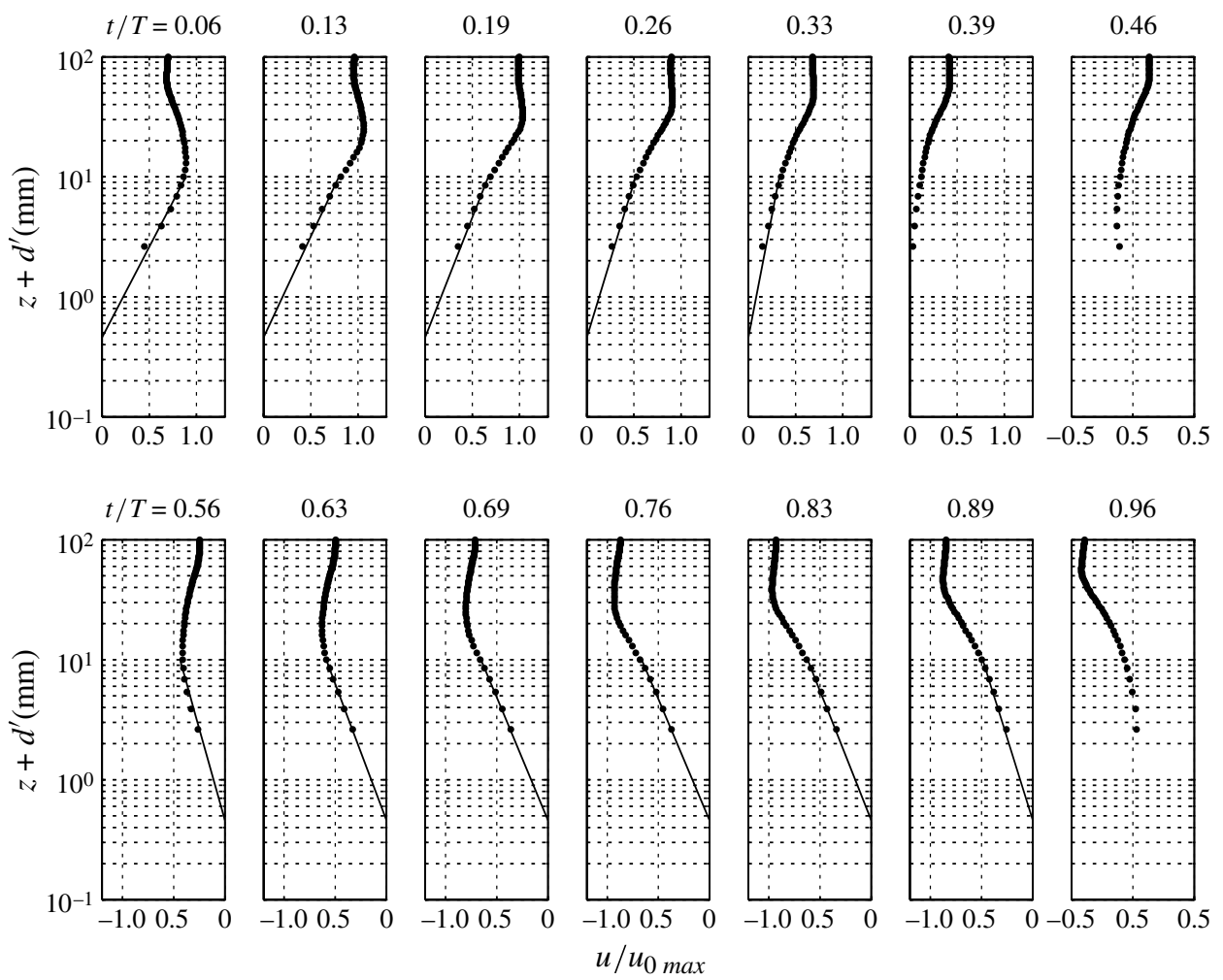

FIGURE 18. Example log-law fits. Experiment S757012g $(\beta=0.75, T=7 \mathrm{~s}$, gravel-rough bed, $\left.k_{s}=13.79 \mathrm{~mm}, d^{\prime}=1.64 \mathrm{~mm}\right)$.

agreement with values of $k_{s}=(2-4) d_{50}$ and $d^{\prime}=(0.15-0.35) d_{50}$ typical of steady flow. For the sand-rough bed, $d^{\prime}=0.25 d_{50}$ and $k_{s}=2.38 d_{50}$ were obtained, the latter with standard deviation $1.02 d_{50}$, which to some extent reflects the uncertainty in accurately determining the $z=0$ level for the sand-rough bed because of the larger ratio of pixel size to $d_{50}$. Additionally, because the boundary layer is much thinner in the case of the sand-rough bed, there are consequently fewer data points to which (6.1) can be fitted. The values of $k_{s}$ in table 1 correspond to $k_{s}=2.44 d_{50}=13.79 \mathrm{~mm}$ for the gravel-rough bed and $k_{s}=2.38 d_{50}=1.09 \mathrm{~mm}$ for the sand-rough bed. With $z_{0}$ and $d^{\prime}$ established for each bed type, the intra-wave friction velocity, $u_{*}(t)$, was obtained by fitting (6.1) to the instantaneous velocity profiles for each experiment. Figure 18 shows examples of the logarithmic fit for experiment S757012g. The fit was accepted only when it was based on three or more measurement points in the profile and the correlation coefficient between data and fit exceeded 0.95 .

Figure 19(a-d) shows the log-law-based bed shear stress for all 12 experiments. As before, each sub-figure contains results for the three values of $\beta$ (nominally 0.5 , 0.6 and 0.75 ) for the same $T, u_{0 \max }$ and bed type. As expected, the bed shear stress shows increasing asymmetry between the two half-cycles as the acceleration skewness increases. Higher acceleration during the positive flow results in higher peak bed shear stress. The peak shear stress shifts earlier in phase, in accordance with the shift of the maximum free-stream velocity with increasing $\beta$. During negative flow, higher bed shear stress again occurs after the largest acceleration, which now results in lower 

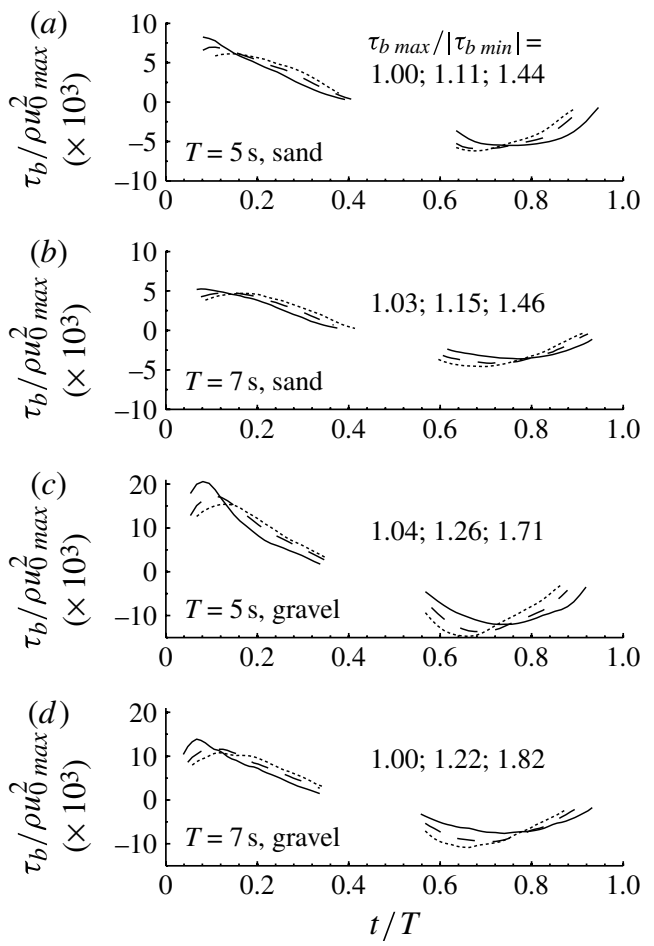

(e)

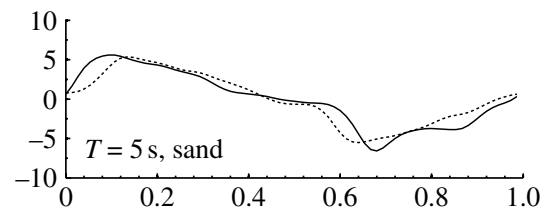

$(f)$

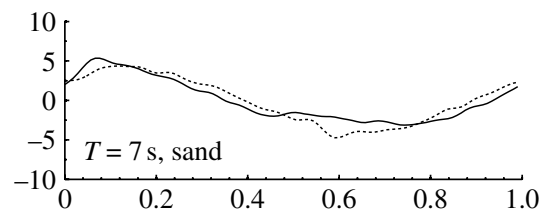

$(g)$

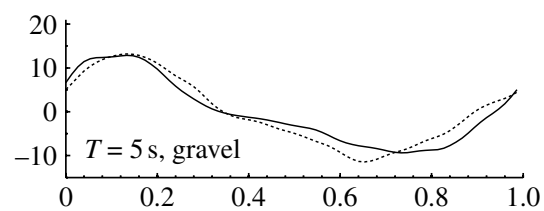

(h)

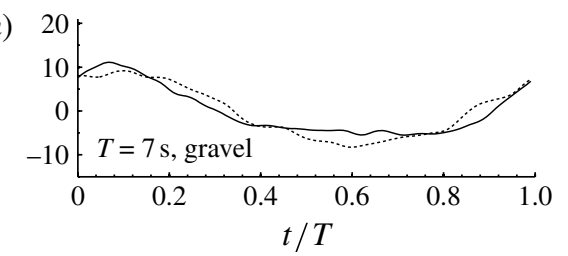

FIGURE 19. $(a-d)$ Bed shear stress obtained from (6.1). $(e-h)$ Bed shear stress obtained from (6.2). $\beta \approx 0.5$ (dotted line); $\beta \approx 0.6$ (dashed line); $\beta \approx 0.75$ (solid line). The solid line in $(d)$ corresponds to the profiles shown in figure 18 .

bed shear stress up to the moment of maximum flow with increasing values of $\beta$. Consequently, for given $\beta>0.5$ distinct asymmetry occurs in the bed shear stress between the positive and negative half-cycles. In each plot is listed, for increasing $\beta$, the ratio of maximum positive to maximum negative bed shear stress $\tau_{b \max } /\left|\tau_{b \text { min }}\right|$. For $\beta \approx 0.5, \tau_{b \max } /\left|\tau_{b \min }\right| \approx 1.0$; for $\beta \approx 0.75, \tau_{b \max } /\left|\tau_{b \min }\right|$ is as high as 1.8 .

\subsection{Momentum integral}

The shear stress at elevation $z=z_{1}$ can be estimated by integration of the momentum equation for one-dimensional horizontally uniform oscillatory boundary layer flow as follows (e.g. Jonsson \& Carlsen 1976; Sleath 1987):

$$
\tau\left(z_{1}, t\right)=-\rho \int_{z_{1}}^{z_{t o p}}\left(\frac{\partial}{\partial t}\left(u(z, t)-u_{0}(t)\right)\right) \mathrm{d} z,
$$

where $z_{\text {top }}$ is the highest level in the PIV measurement window. Figure $19(e-h)$ shows the time-dependent shear stress extrapolated to $z+d^{\prime}=0$ for the eight experiments with the PIV camera in position 2 (smaller measurement window, see $\$ 2.2$ ). The shear stress obtained from the momentum integral displays a temporal behaviour similar to the bed shear stress obtained from the log-law method and generally indicates that, with increasing acceleration skewness, peak shear stress during positive flow increases and during negative flow it decreases. For most conditions however, especially the gravel bed conditions, the bed shear stress obtained via (6.2) is lower than the bed shear stress obtained from the log-law. This is most likely due to the presence of the vertical velocities, which cause non-uniformity in the horizontal flow; accounting 
for the non-uniformity requires a $\partial(u u) / \partial x$ term to be added within the integral in (6.2). However, it was not possible to evaluate $\partial(u u) / \partial x$ from the present data, because it is subject to significant error since the term requires calculation of small differences between large quantities. Furthermore, the presence of secondary currents in the facility as indicated in $\S 4.5$ would require the three-dimensional momentum equation to be evaluated which is not possible with the present data.

\subsection{Shear stress contributions}

The presence of time-averaged and periodic vertical velocities means that there are additional contributions to shear stress, over and above the viscous and turbulent Reynolds stress. Derivation of the Reynolds equations for combined wave-current flow, whereby the velocity components are decomposed into a mean, a periodic and a fluctuating part $\left(u=\bar{u}+\tilde{u}+u^{\prime}\right.$ and $\left.w=\bar{w}+\tilde{w}+w^{\prime}\right)$ leads to the total periodic shear stress given by Nielsen (1992):

$$
\tilde{\tau}=\rho \nu \frac{\partial \tilde{u}}{\partial z}-\rho \widehat{u^{\prime} w^{\prime}}-\rho \bar{u} \tilde{w}-\rho \tilde{u} \bar{w}-\rho \tilde{u} \tilde{w},
$$

where the first term on the right-hand side is the viscous stress, the second is the turbulent stress, the third and fourth terms describe convective transfer of momentum and the last term is due to correlations between the periodic velocity components. Figure 20 shows time series of the most dominating contributions to $\tilde{\tau}$, namely $\widehat{u^{\prime} w^{\prime}}$, $\tilde{u} \bar{w}$ and $\tilde{u} \tilde{w}$; the contributions of the viscous stress and $\bar{u} \tilde{w}$ are negligibly small in comparison and are therefore not shown. For the gravel bed condition (figure 20a), near the bed, the total stress, $\tilde{\tau}$, is composed of the last three terms in (6.3), each component approximately equal in phase and magnitude and consequently contributing equally to the total stress. Owing to the asymmetry of the individual components, the total oscillatory stress shows a distinct asymmetry between the two half-cycles, with a higher stress during the positive half-cycle compared with the negative half-cycle, similar to the asymmetry observed in the bed shear stress from the log-law and the shear stress obtained from the momentum integral. The main difference between the sand and gravel bed conditions is the contribution of $-\rho \bar{w} \tilde{u}$ near the bed, which is only large for the gravel bed condition due to the relatively large negative $\bar{w}$ velocities measured close to the gravel bed (cf. figure 12b), and which are most likely the result of our limited averaging window. At $z=8.2 \mathrm{~mm}$ the magnitude of $-\rho \bar{w} \tilde{u}$ is decayed because of small $\bar{w}$, but at higher elevations it is seen to increase owing to the secondary $\bar{w}$ velocities above this level, which is also the case for the coarse sand condition.

The agreement between $\tilde{\tau}$ at the lowest elevations in figure 20 and the bed shear stress for the same conditions in figure 19 is remarkably good. It appears however that in terms of the magnitude, $\tilde{\tau}$ is generally lower than the shear stresses presented in figure 19, as was also the case for the other experiments, which are not presented here for brevity. The fact that $\tilde{\tau}$ close to the bed is consistently lower than the bed shear stress estimates in figure 19 could be due to the presence of significant form-induced stresses which are not captured in (6.3), but require estimation of the the spatial- and time-averaged Reynolds equations (Coleman, Nikora \& Schlicke 2008).

\subsection{Friction factor}

For the present conditions separate friction factors for the positive and negative flow half-cycles are defined as follows:

$$
f_{w c}=\frac{2 u_{* \max }^{2}}{u_{0 \max }^{2}},
$$




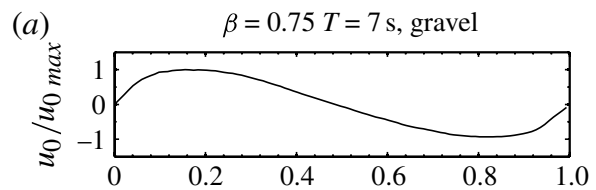

(b)
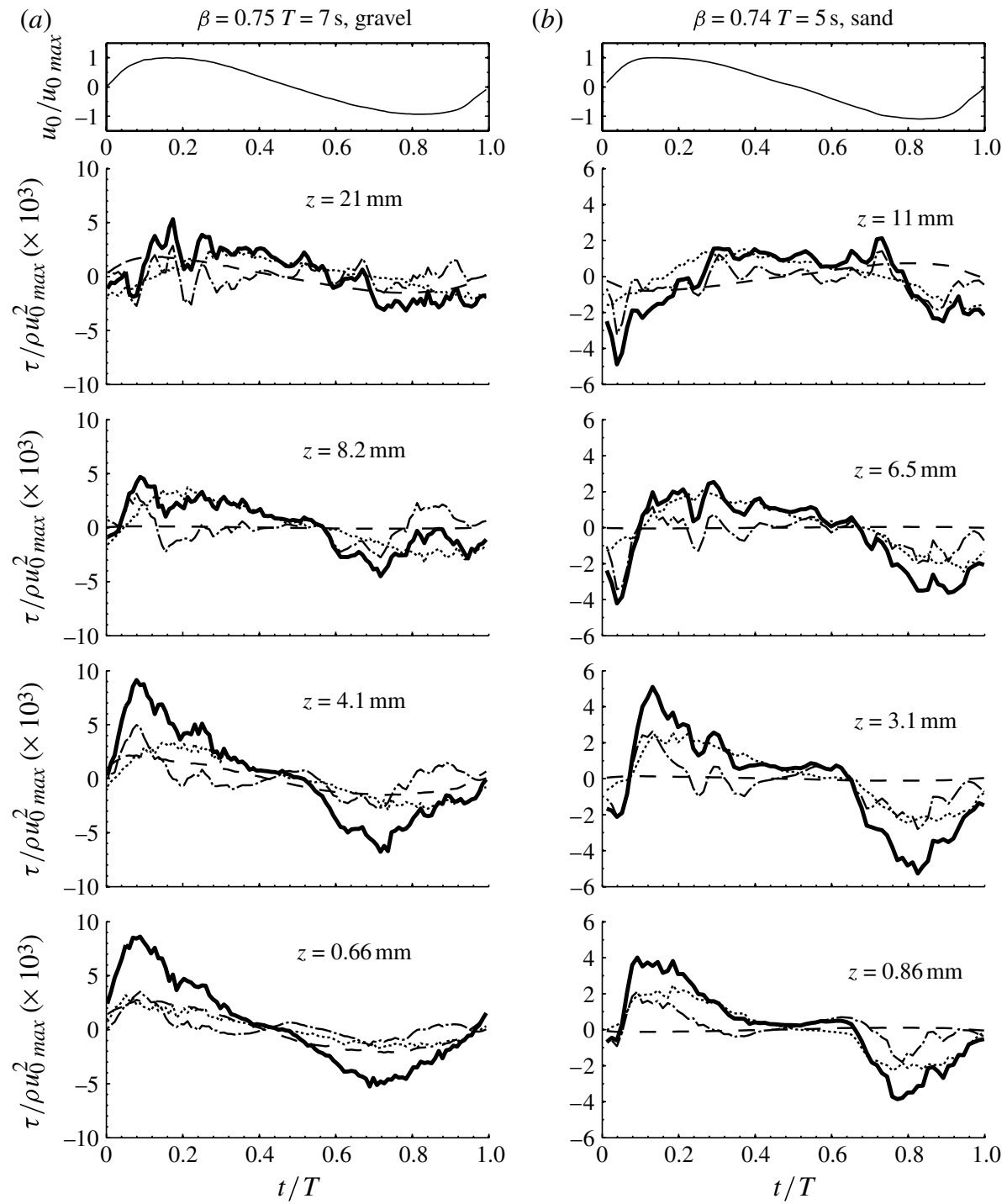

FIGURE 20. Time series of the total shear stress $\tilde{\tau}$ (solid), turbulent stress $-\rho \widehat{u^{\prime} w^{\prime}}$ (dotted), $-\rho \tilde{u} \bar{w}$ (dashed) and $-\rho \tilde{u} \tilde{w}$ (dash-dot), for two experiments: (a) S757012g (b) S755010c. Results are shown at four elevations above the bed in each case.

$$
f_{w t}=\frac{2 u_{* \min }^{2}}{u_{0 \min }^{2}}
$$

with $u_{*}$ obtained from (6.1). Figure 21 shows the friction factors for the present experiments alongside previous results for sinusoidal flow and Swart's (1974) frequently used empirical equation. For the present conditions, the positive half-cycle friction factor, $f_{w c}$, is plotted against $a_{c} / k_{s}$ and similarly $f_{w t}$ is plotted against $a_{t} / k_{s}$ where $a_{t}=a 2 T_{a t} / T_{t}$ with the periods $T_{a t}$ and $T_{t}$ as outlined in figure 2 . In this case the calculation of $a$ for each half-cycle takes account of the fact that for $\beta$ increasing above 0.5 the time from zero velocity to peak positive velocity decreases and the time from zero to peak negative velocity increases (e.g Silva et al. 2006; Gonzalez- 


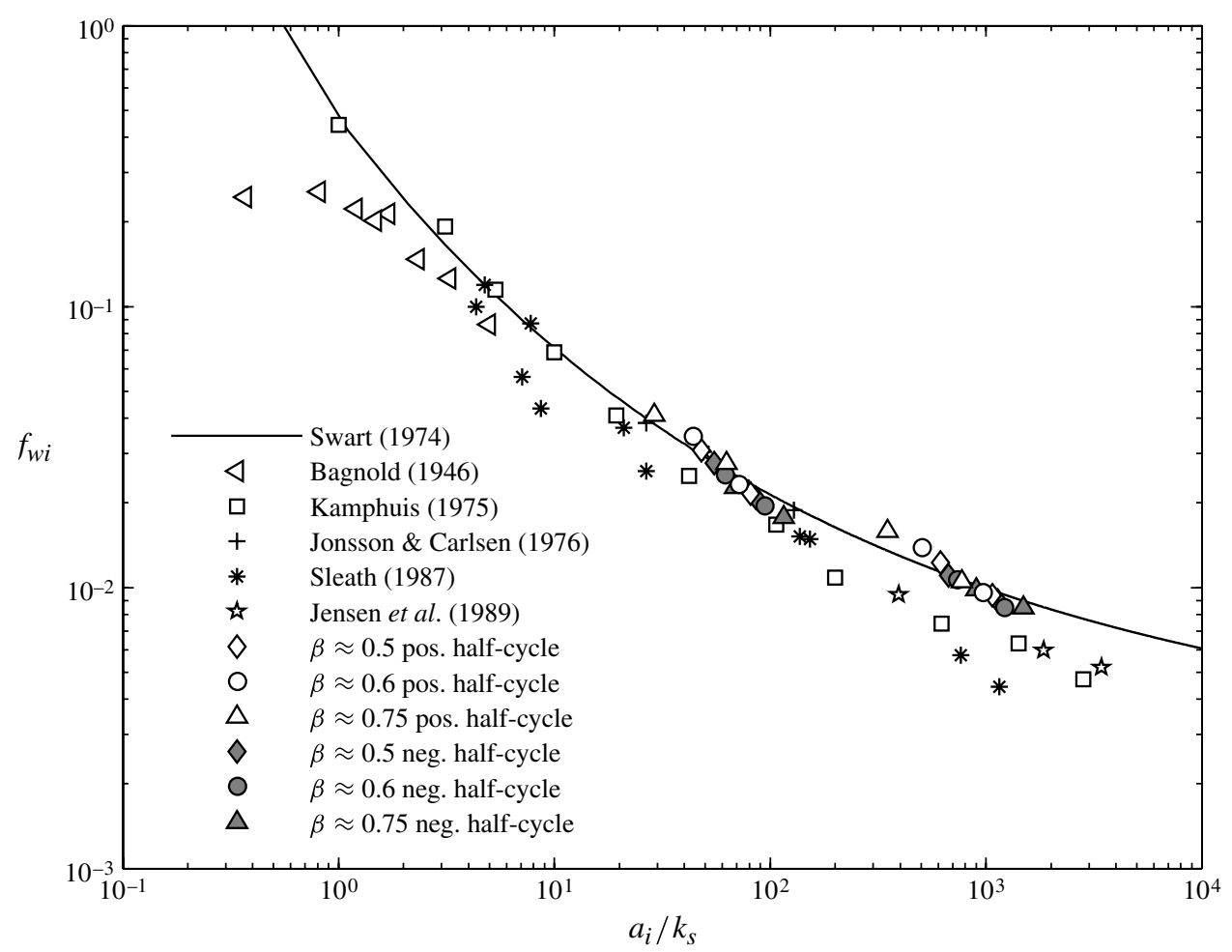

FIGURE 21. Wave friction factor $f_{w}$ versus relative roughness. Here $a_{i}$ corresponds to $a_{c}$ for the positive half-cycle and to $a_{t}$ for the negative half-cycle. The other studies shown all involved flows without acceleration skewness for which $a_{c}=a_{t}=a$.

Rodriguez \& Madsen 2007). Plotted in this way, the half-cycle friction factors from the present experiments show very good agreement with Swart (1974). Note that for all the previous studies in figure $21, \beta=0.5$ which results in $a_{c}=a_{t}=a$. If the present results with acceleration-skewed flows were plotted against $a / k_{s}$, the results would still be in good agreement with Swart, but with $f_{w p}$ and $f_{w n}$ lying either side of the Swart (1974) curve. These results confirm that the approaches of Silva et al. (2006) and Gonzalez-Rodriguez \& Madsen (2007), whereby the friction factor for each half-cycle is based on an equivalent sinusoidal orbital amplitude, is a promising, practical approach to determine bed shear stress for acceleration-skewed flow.

\section{Conclusions}

Experiments have been conducted in a large oscillatory flow tunnel to investigate the effects of acceleration skewness on oscillatory boundary layer flow. The experiments involved two types of bed roughness and a range of high-Re oscillatory flow conditions varying from sinusoidal to highly acceleration-skewed. The detailed velocity measurements obtained using PIV are supported through good agreement with LDA point-velocity measurements. The most significant results from the study are summarized as follows.

(a) The effect of acceleration skewness is clearly seen in the boundary layer velocity profiles, with higher accelerations resulting in higher peak near-bed velocities and higher near-bed velocity gradients. 
(b) Harmonic analysis of horizontal velocity shows that the relative amplitude of the second harmonic (compared to the first-harmonic amplitude) remains reasonably constant throughout the boundary layer, while the third harmonic grows in relative importance towards the bed; strong oscillatory behaviour of the phase of the third harmonic suggests strong nonlinear interactions in the boundary layer.

(c) Horizontal velocity near the bed leads the free-stream horizontal velocity. The phase lead, $\phi$, of the first harmonic agrees very well with phase lead values from previous studies for sinusoidal flow, and confirms the existence of a logarithmic relationship between $\phi$ and $a / k_{s}$. The phase lead appears to decrease slightly with increasing acceleration skewness, $\beta$. As the bed is approached, the positive-tonegative flow reversal occurs sooner with increasing $\beta$ and the negative-to-positive flow reversal occurs later with increasing $\beta$, which results in a shorter-duration positive half-cycle compared to the negative half cycle.

(d) With increasing $\beta$, the boundary layer at maximum positive velocity is thinner compared to the boundary layer at maximum negative velocity. When scaled with the appropriate orbital excursion, the boundary layer thickness at maximum velocity depends only on relative roughness as per (4.1), in agreement with previous results for sinusoidal flow.

(e) Acceleration skewness generates a time-averaged residual horizontal flow in the boundary layer, with direction against the direction of highest acceleration and with magnitude increasing with increasing $\beta$.

( $f$ ) Turbulence is generated at the bed and diffuses upwards; turbulence intensity increases as the flow accelerates after flow reversal, is maximum at around maximum free-stream velocity and decays as the flow decelerates. Acceleration skewness leads to a more rapid rate of increase in turbulence intensity and a somewhat higher peak intensity during the positive half-cycle compared to the negative half-cycle. Residual turbulence at flow reversal (generated during the previous half cycle) is greater at the negative-to-positive flow reversal than at the positive-to-negative reversal because of the difference in the duration of flow deceleration. Despite substantial differences in intra-wave turbulence intensity for varying $\beta$, time-averaged turbulence intensity is independent of $\beta$.

(g) Similar to turbulence intensity, the intra-wave turbulent (Reynolds) stress depends on $\beta$. Highest Reynolds stress generally coincides with the high-acceleration positive flow. Time-averaged Reynolds stress profiles show a slight dependence on $\beta$, with higher Reynolds stress occurring for larger $\beta$.

(h) A logarithmic layer exists during large parts of the flow cycle, in agreement with previous research results. As expected, bed shear stress estimates obtained from the law of the wall show increasing asymmetry between the two flow half-cycles with increasing $\beta$. The peak positive bed shear stress increases and the peak negative bed shear stress decreases as $\beta$ increases. The ratio of peak positive to peak negative bed shear stress is larger for larger roughness.

(i) Bed shear stress estimated from the momentum integral approach shows similar asymmetry between the flow half-cycles with increasing $\beta$ as that seen in the log-law-based estimates and agreement between bed shear stress magnitudes from the two methods is generally good.

(j) Turbulent Reynolds stress is much less than the shear stress obtained from the momentum integral approach. The measurements show that significant phaseaveraged vertical velocities exist near the bed throughout the flow cycle, most likely associated with periodic formation and ejection of vortices around the roughness grains. These periodic vertical velocities lead to an additional shear 
stress, $-\rho \tilde{u} \tilde{w}$, which, near the bed, is at least as large as the turbulent Reynolds stress.

(k) When account is taken of the 'equivalent' sinusoidal amplitude of water particle displacement, half-cycle wave friction factors show very good agreement with results from previous studies and with Swart's (1974) empirical equation, confirming the viability of recently proposed practical approaches for calculating bed shear stress in acceleration skewed flow.

The experimental data forming the basis of this paper are available by request to the authors.

This work has been carried out within the project SANTOSS ('SANd Transport in OScillatory flows in the Sheet-flow regime') funded by the UK's EPSRC (GR/T28089/01) and the Dutch research organisation STW (TCB.6586). The authors acknowledge the support of the technical staff at the University of Aberdeen, especially Fluids Laboratory technician Rab Fraser. Useful comments from the reviewers have helped improve the manuscript.

\section{REFERENCES}

VAn Der A, D. A., O’Donoghue, T. \& Ribberink, J. S. 2010 Measurements of sheet flow transport in acceleration-skewed oscillatory flow and comparison with practical formulations. Coast. Engng 57, 331-342.

Bucchave, P., George, W. K. \& Lumley, J. L. 1979 The measurement of turbulence with the laser-doppler anemometer. Annu. Rev. Fluid Mech. 11, 443-503.

Chen, D., Chen, C., Tang, F.-E., Stansby, P. \& Li, M. 2007 Boundary layer structure of oscillatory open-channel shallow flows over smooth and rough beds. Exp. Fluids 42 , 719-736.

Coleman, S. E., Nikora, V. I. \& Schlicke, T. 2008 Spatially-averaged oscillatory flow over a rough bed. Acta Geophys. 56, 698-733.

Conley, D. C. \& InMAn, D. L. 1994 Ventilated oscillatory boundary layers. J. Fluid Mech. 273, 261-284.

Cox, D. T., Kobayashi, N. \& OKayasu, A. 1996 Bottom shear stress in the surf zone. J. Geophys. Res. 101 (C6), 14337-14348.

DAVIES, A. G. \& LI, Z. 1997 Modelling sediment transport beneath regular symmetrical and asymmetrical waves above a plane bed. Cont. Shelf Res. 17, 555-582.

DAVIES, A. G. \& Villaret, C. 1997 Oscillatory flow over rippled bed: boundary layer structure and wave-induced Eulerian drift. In Gravity Waves in Water of Finite Depth, Advances in Fluid Mechanics (ed. J. Hunt), pp. 215-254. Computational Mechanics Publications.

Dixen, M., Hatipoglu, F., Sumer, B. M. \& Fredsøe, E. J. 2008 Wave boundary layer over a stone-covered bed. Coast. Engng 55, 1-20.

Dohmen-Janssen, C. M., Kroekenstoel, D. F., Hassan, W. N. \& Ribberink, J. S. 2002 Phase lags in oscillatory sheet flow: experiments and bed load modelling. Coast. Engng 46, 61-87.

Elfrink, B., Hanes, D. M. \& Ruessink, B. G. 2006 Parameterization and simulation of near bed orbital velocities under irregular waves in shallow water. Coast. Engng 53, 915-927.

Elgar, S., Gallagher, E. L. \& GuZA, R. T. 2001 Nearshore sandbar migration. J. Geophys. Res. 106 (C6), 11623-11627.

Foster, D. L., Guenther, R. A. \& Holman, R. A. 1999 An analytic solution to the wave bottom boundary layer governing equation under arbitrary wave forcing. Ocean Engng 26, 595-623.

Fredsøe, J. \& Deigaard, R. 1992 Mechanics of Coastal Sediment Transport. World Scientific.

Fredsøe, J., Sumer, B. M., Laursen, T. S. \& Pedersen, C. 1993 Experimental investigation of wave boundary layers with a sudden change in roughness. J. Fluid Mech. 252, 117-145. 
Fredsøe, J., Sumer, B. M., Kozakiewicz, A., Chua, L. H. C. \& Deigaard, R. 2003 Effect of externally generated turbulence on wave boundary layer. Coast. Engng 49, 155-183.

Fuhrman, D. R., FredsøE, J. \& Sumer, B. M. 2009 Bed slope effects on turbulent wave boundary layers: 2. Comparison with skewness, asymmetry and other effects. J. Geophys. Res. 114, C03025.

Gonzalez-Rodriguez, D. \& Madsen, O. S. 2007 Seabed shear stress and bedload transport due to asymmetric and skewed waves. Coast. Engng 54, 914-929.

GRASS, A. J. 1971 Structural features of turbulent flow over smooth and rough boundaries. J. Fluid Mech. 50, 233-255.

Holmedal, L. E. \& MYrhaug, D. 2006 Boundary layer flow and net sediment transport beneath asymmetrical waves. Cont. Shelf Res. 26 (2), 252-268.

Hsu, T.-J. \& Hanes, D. M. 2004 Effects of wave shape on sheet flow sediment transport. J. Geophys. Res. 109, C05025.

Jensen, B. L., Sumer, B. M. \& FredsøE, J. 1989 Turbulent oscillatory boundary layers at high reynolds numbers. J. Fluid Mech. 206, 265-297.

Jonsson, I. G. 1963 Proc. 10th Congress IAHR, London, vol. 1, pp. 85-92.

Jonsson, I. G. 1980 A new approach to oscillatory rough turbulent boundary layers. Ocean Engng 7, 109-152.

Jonsson, I. G. \& CARLSEn, N. A. 1976 Experimental and theoretical investigations in an oscillatory turbulent boundary layer. J. Hydraul Res. 14, 45-60.

KeAne, R. D. \& AdRian, R. D. 1992 Theory of cross-correlation analysis of PIV images. Appl. Sci. Res. 49, 191-215.

Keiller, D. C. \& Sleath, J. F. A. 1976 Velocity measurements close to a rough plate oscillating in its own plane. J. Fluid Mech. 73, 673-691.

Kemp, P. H. \& Simons, R. R. 1982 The interaction of waves with a turbulent current: waves propagating with the current. J. Fluid Mech. 116, 227-250.

Kemp, P. H. \& Simons, R. R. 1983 The interaction of waves with a turbulent current: waves propagating against the current. J. Fluid Mech. 130, 73-89.

Krstic, R. V. \& FERnAndo, H. J. S. 2001 The nature of rough-wall oscillatory boundary layers. J. Hydraul. Res. 39, 655-666.

Mirfenderesk, H. \& Young, I. R. 2003 Direct measurements of the bottom friction factor beneath surface gravity waves. Appl. Ocean Res. 25, 269-287.

Nielsen, P. 1992 Coastal Bottom Boundary Layers and Sediment Transport. World Scientific.

O'Donoghue, T. \& Wright, S. 2004 Flow tunnel measurements of velocities and sand flux in oscillatory sheet flow for well-sorted and graded sands. Coast. Engng 51, 1163-1184.

Ranasoma, K. \& Sleath, J. 1992 Velocity measurements close to rippled beds. In Proc. 23rd Intl Conf. on Coastal Engng (ed. Billy L. Edge), pp. 2383-2396. ASCE.

RibberinK, J. S. \& Al-SAlEM, A. A. 1994 Sediment transport in oscillatory boundary layers in cases of rippled beds and sheet flow. J. Geophys. Res. 99 (C6), 12707-12727.

Ribberink, J. S. \& Al-Salem, A. A. 1995 Sheet flow and suspension of sand in oscillatory boundary layers. Coast. Engng 25, 205-225.

Silva, P. A., Temperville, A. \& Seabra-Santos, F. 2006 Sand transport under combined current and wave conditions: a semi-unsteady, practical model. Coast. Engng 53, 897-913.

SLEATH, J. F. A. 1970 Velocity measurements close to the bed in a wave tank. J. Fluid Mech. 42, 111-123.

Sleath, J. F. A. 1987 Turbulent oscillatory flow over rough beds. J. Fluid Mech. 182, 369-409.

Soulsby, R. L. 1983 The bottom boundary layer of shelf seas. In Physical Oceanography of Coastal and Shelf Seas (ed. B. Johns), pp. 189-266. Elsevier.

Sumer, B. M., LAursen, T. S. \& Fredsøe, J. 1993 Wave boundary layers in a convergent tunnel. Coast. Engng 20, 317-342.

Swart, D. H. 1974 Offshore sediment transport and equilibrium beach profiles. In Publication No. 131. Delft Hydraulics Laboratory.

Trowbridge, J. \& Madsen, O. S. 1984 Turbulentwave boundary layers 2. Second-order theory and mass transport. J. Geophys. Res. 89 (C5), 7999-8007. 
Watanabe, A. \& SATO, S. 2004 A sheet-flow transport rate formula for asymmetric forward-leaning waves and currents. In Proc. 29th Intl Conf. on Coastal Engng (ed. J. McKee Smith). World Scientific.

Westergatrd, C. H., Madsen, B. B., Marassi, M. \& Tomasini, E. P. 2003 Accuracy of PiV signals in theory and practice. In 5th Intl Symp. on Particle Image Velocimetry, Busan, Korea, Paper 3301.

WeSTERWEel, J. 1994 Efficient detection of spurious vectors in particle image velocimetry data. Exp. Fluids 16, 236-274.

Westerweel, J., Dabiri, D. \& Gharib, M. 1997 The effect of a discrete window offset on the accuracy of cross-correlation analysis of digital piv recordings. Exp. Fluids 23, 20-28. 